\title{
A MODEL PROBLEM FOR BOUNDARY LAYERS OF THIN ELASTIC SHELLS
}

\author{
Philippe Karamian ${ }^{1}$, JACQUeline SANCheZ-Hubert ${ }^{1,2}$ \\ AND Évarisite SANChez PALENCIA ${ }^{2}$
}

\begin{abstract}
We consider a model problem (with constant coefficients and simplified geometry) for the boundary layer phenomena which appear in thin shell theory as the relative thickness $\varepsilon$ of the shell tends to zero. For $\varepsilon=0$ our problem is parabolic, then it is a model of developpable surfaces. Boundary layers along and across the characteristic have very different structure. It also appears internal layers associated with propagations of singularities along the characteristics. The special structure of the limit problem often implies solutions which exhibit distributional singularities along the characteristics. The corresponding layers for small $\varepsilon$ have a very large intensity. Layers along the characteristics have a special structure involving subspaces; the corresponding Lagrange multipliers are exhibited. Numerical experiments show the advantage of adaptive meshes in these problems.
\end{abstract}

Résumé. Nous considérons un problème modèle (avec coefficients constants et géométrie simplifiée) pour l'étude des couches limites qui apparaissent en théorie des coques élastiques minces lorsque l'épaisseur relative $\varepsilon$ tend vers zéro. Pour $\varepsilon=0$, notre problème est parabolique, c'est donc un problème modèle pour les surfaces développables. Les couches limites le long et transversalement aux caractéristiques ont des structures très différentes. Il apparaît aussi des couches internes associées à la propagation des singularités le long des caractéristiques. Dans certains cas, à cause de sa structure particulière, le problème limite a des solutions qui présentent des singularités faisant intervenir des distributions le long des caractéristiques. Pour $\varepsilon$ petit, les couches correspondantes sont de très grande intensité. Les couches le long des caractéristiques ont une structure particulière incluant des sousespaces ; les multiplicateurs de Lagrange correspondants sont mis en évidence. Les calculs numériques montrent l'avantage de l'utilisation de maillages adaptés dans ce type de problèmes.

Mathematics Subject Classification. 73K15, 35B25.

Received: April 30, 1999.

\section{INTRODUCTION}

This paper is devoted to a model problem for the boundary and internal layers in shells so we first recall some features of thin shell theory. For generalities on shells the reader may refer to $[1,4,7,18]$. Roughly speaking, a shell is described by a thin body, of thickness $2 \varepsilon$, close to a surface $S$ the boundary of which is submitted to some kinematic conditions. The mechanical behavior is described by two bilinear forms $a(u, v)$ and $\varepsilon^{2} b(u, v)$, associated with the deformations of the intrinsic metrics and the variations of curvature, which are called the

\footnotetext{
Keywords and phrases. Shells, boundary layers, singular perturbation.

1 Laboratoire de Mécanique, Université de Caen, Boulevard Maréchal Juin, 14032 Caen Cedex, France.

2 Laboratoire de Modélisation en Mécanique, Université Paris VI, 4 place Jussieu, 75252 Paris Cedex 05, France.

e-mail: sanchez@lmm.jussieu.fr
} 
membrane form and the flexion form respectively. Note that the second one involves a factor $\varepsilon^{2}$, accounting for the small rigidity of a thin body to flexion. This fact entails very specific asymptotic properties for small $\varepsilon$, a part of them will be described hereafter, we refer mainly to $[5,7,19]$ for more details.

In the sequel, we only consider the so called "inhibited shells", i.e. such that the surface $S$ along with the kinematic boundary conditions is geometrically rigid. The corresponding system is of the form $A+\varepsilon^{2} B$, where $B$ is elliptic but $A$ is of the same type as the points of the surface $S$, i.e. elliptic, parabolic or hyperbolic at elliptic, parabolic or hyperbolic points of the surface. In addition the order of derivation in $B$ is higher than in $A$ so that as $\varepsilon$ tends to zero there is a singular perturbation phenomenon. In the sequel, we shall focus on the case when $A$ is uniformly parabolic which corresponds to developpable surfaces. The limit process $\varepsilon \searrow 0$ is very singular, as it goes from a higher order elliptic system to a parabolic system. For $\varepsilon>0$, the energy space $V$ is chosen so that $a+\varepsilon^{2} b$ be continuous and coercive on it, whereas the limit problem involves a new energy space $V_{a}$ such that the form $a$ is continuous and coercive on it. In fact, $V_{a}$ is the completion of $V$ with the norm $\sqrt{a(\cdot, \cdot)}$ (note that, it is a norm as a consequence of the hypothesis of inhibition).

Obviously, the space $V_{a}$ contains functions which are less smooth than the functions of $V$. Consequently, the solutions $u^{\varepsilon}$ belong to $V$ but the limit as $\varepsilon$ tends to zero is a less smooth function. As a consequence, $u^{\varepsilon}$ for small $\varepsilon$ exhibits "boundary layers" i.e. narrow regions where the gradient grows very quickly. In fact, there is another, even more important reason for the presence of boundary layers. Of course, as $V \subset V_{a}$, the dual spaces verify $V_{a}^{\prime} \subset V^{\prime}$ so that the admissible forces which are in $V^{\prime}$ for $\varepsilon>0$ may not be admissible for the limit problem. In this case, the energy of $u^{\varepsilon}$ tends to infinity, as we prove in Section 2.1. The corresponding solution of the limit problem is out of $V_{a}$ and exhibits distributional singularities so that $u^{\varepsilon}$ involves boundary layers of large intensity (see also [11] in this context). It is to be mentioned that this situation is very common in shell theory where the space $V_{a}$, which depends on the geometry, is often very large and consequently $V_{a}^{\prime}$ is a very small space. The most typical example of such a situation is given by the so called "sensitive shells" for which the space $V_{a}^{\prime}$ is so small that is does not contain the space $\mathcal{D}$ of test functions of distributions [12, 13]. Obviously, for sensitive shells almost any loading $f$ is out of $V_{a}^{\prime}$ but, even for non-sensitive shells "very usual" loadings may be out of $V_{a}^{\prime}$. For instance, in ruled surfaces with a free boundary along a generator, any loading not vanishing on that generator is out of $V_{a}^{\prime}$ (see [18], Sects. VII.2.4 and VII.4.2 as well as Sect. 2.2 of this paper for the model problem).

It should be emphasized that the non-smoothness of solutions of the limit problem has important consequences on the finite element computations of $u^{\varepsilon}$ for small $\varepsilon$. It is not hard to prove (see [6] for instance) that when $f \notin V_{a}^{\prime}$ the convergence of the finite element approximations $u_{k}^{\varepsilon}$ to $u^{\varepsilon}$ cannot be uniform with respect to $\varepsilon \in\left(0, \varepsilon_{0}\right)$ with values in $V_{a}$ (and then also in any "smaller" space!). In other words, the smaller $\varepsilon$ is the smaller $h$ must be chosen in order to get a good approximation. We may refer to $[8,10]$ for these features. As a result, the situation in the present case, of inhibited shells, is analogous to that of non-inhibited ones where the non-uniformity of the convergence is a consequence of the phenomenon of locking which appears for any conformal approximation with piecewise polynomial finite elements [3].

Up to our knowledge, very little is known about boundary layers of shells. They are called "edge effects" in $[7,14]$ which are mainly concerned with layers transversal to the characteristics. Specific solutions may perhaps be found in the impressive catalogue of analytical solutions of Rutten [16] but their utilisation in specific problems is not evident. So, in this paper, we consider a model problem for the above mentioned shell problems in the case when the limit operator $A$ is parabolic. The model has constant coefficients and involves two unknowns $u_{1}$ and $u_{2}$; the first one plays the role of the two tangential components of the displacement vector in shells and the second is analogous to the normal component.

The paper is organized as follows: the model problem $P(\varepsilon)$ is presented hereafter at the end of this introduction (see (1.1)-(1.5)). The limit problem $P(0)$ is addressed in Section 2 which includes properties of the asymptotic process $\varepsilon \searrow 0$ (see Sect. 2.1) and a criterion for $f \in V_{a}^{\prime}$ which is an adaptation of a criterion of sensitivity [13] (see Sect. 2.2). The scaling for the layers, either along or across the characteristics is obtained in Section 3 by a method based on asymptotic trends of exponential solutions. We point out that this method was used in another context in [15]. Boundary layer along a clamped characteristic is considered in Section 4. 
The specific equations and boundary conditions are obtained by formal asymptotic expansions directly from the variational formulation. The analogous problem for a free boundary is handled in Section 5 , two cases appear according to the loading vanishes or not on the boundary. In the second case, the intensity of the layer is very much larger because $f \notin V_{a}^{\prime}$. Section 6 is devoted to the case of internal layers along the characteristics, the limit of which is the phenomenon of propagation of singularities. The structure of the characteristic layers involves a special structure involving subspaces. The corresponding Lagrange multiplier is considered in Section 7 . The more classical case of layers transversal to the characteristics is addressed in Section 8. In Section 9, we prove that the limit as $\varepsilon \searrow 0$ of the internal layers are the solutions in the distributions sense of the limit problem which were given in Section 2.4 for $f \notin V_{a}^{\prime}$. Section 10 contains numerical experiments with finite element approximations, mainly for $\varepsilon=10^{-3}$. The advantage of using meshes adapted to the specific features of the layers is shown. The principal results, thicknesses and intensities of the layers in different cases are summed up in a table in Section 11.

The model problem $P(\varepsilon)$ is defined as follows. Let us consider the domain $\Omega=(0, \pi) \times(0, \pi)$ of the plane $x=\left(x_{1}, x_{2}\right)$. The boundary $\partial \Omega$ is composed of two parts $\Gamma_{0}$ and $\Gamma_{1}$ which are respectively the "clamped " part and the "free" part, they will be precisely defined later. Several choices will be done but, in any case, $\Gamma_{0}$ is made of whole sides and always contains two adjacent sides.

The configuration space $V$ is a space of elements $v=\left(v_{1}, v_{2}\right)$ satisfying the boundary conditions:

$$
v_{1}=v_{2}=\partial_{n} v_{2}=0 \text { on } \Gamma_{0}
$$

where $\partial_{n}$ denotes the normal derivative to the boundary, more precisely

$$
V=\left\{v \in H^{1}(\Omega) \times H^{2}(\Omega) ; v \text { satisfies }(1.1)\right\}
$$

We consider the two bilinear forms

$$
\begin{aligned}
a(u, v) & =\int_{\Omega}\left[\left(\partial_{1} u_{1}\right)\left(\partial_{1} v_{1}\right)+\left(\partial_{2} u_{1}-u_{2}\right)\left(\partial_{2} v_{1}-v_{2}\right)\right] \mathrm{d} x \\
b(u, v) & =\int_{\Omega} \sum_{|\alpha| \leq 2} \partial_{\alpha} u_{2} \partial_{\alpha} v_{2} \mathrm{~d} x \equiv\left(u_{2}, v_{2}\right)_{2}
\end{aligned}
$$

Let us denote by $f$ a given element of $V^{\prime}$ (the dual of $V$ ), the problem $P(\varepsilon)$ writes:

$$
\left\{\begin{array}{l}
\text { Find } u^{\varepsilon} \text { such that } \forall v \in V \\
a\left(u^{\varepsilon}, v\right)+\varepsilon^{2} b\left(u^{\varepsilon}, v\right)=\langle f, v\rangle_{V^{\prime} V}
\end{array}\right.
$$

From the previous hypotheses, it immediately follows that the problem $P(\varepsilon)$ with fixed $\varepsilon>0$ is continuous and coercive on $V$, so that the existence and uniqueness of the solution is ensured by the Lax Milgram theorem. Clearly the coerciveness constant is of order $\varepsilon^{2}$ so that it vanishes as $\varepsilon \searrow 0$.

This problem is somewhat classical. It is equivalent to the system of equations

$$
\left\{\begin{array}{l}
-\Delta u_{1}^{\varepsilon}+\partial_{2} u_{2}^{\varepsilon}=f_{1} \\
-\partial_{2} u_{1}^{\varepsilon}+u_{2}^{\varepsilon}+\varepsilon^{2}\left(\Delta^{2} u_{2}^{\varepsilon}-\Delta u_{2}^{\varepsilon}+u_{2}^{\varepsilon}\right)=f_{2}
\end{array}\right.
$$

with the principal boundary conditions $(1.1)$ on $\Gamma_{0}$ and the natural boundary conditions on $\Gamma_{1}$ :

$$
\left\{\begin{array}{l}
\partial_{n} u_{1}^{\varepsilon}-n_{2} u_{2}^{\varepsilon}=F_{1} \\
\varepsilon^{2}\left[\partial_{n} u_{2}^{\varepsilon}-\partial_{n} \Delta u_{2}^{\varepsilon}-\partial_{t}\left(\partial_{n t} u_{2}^{\varepsilon}\right)\right]=F_{2} \\
\varepsilon^{2} \partial_{n n} u_{2}^{\varepsilon}=\mathcal{C}
\end{array}\right.
$$


where $n$ (resp. $t$ ) is the unit outer normal (resp. the unit tangent) to $\Gamma_{1}$ and where

$$
\langle f, v\rangle_{V^{\prime} V}=\int_{\Omega} f_{i} v_{i} \mathrm{~d} x+\int_{\Gamma_{1}}\left(F_{1} v_{1}+F_{2} v_{2}+\mathcal{C} \frac{\partial v_{2}}{\partial n}\right) \mathrm{d} s
$$

in the sequel, we always take $F_{1}=F_{2}=\mathcal{C}=0$ unless the opposite is explicitly said.

Moreover,

$$
v \in V, a(v, v)=0 \Rightarrow v=0
$$

so that

$$
\|v\|_{V_{a}}=(a(v, v))^{\frac{1}{2}}
$$

is a norm on $V$. We denote by $V_{a}$ the completion of $V$ with this norm. We note that (1.9) is analogous to the hypothesis of inhibition in shell theory.

Also we define the limit problem $P(0)$ in variational form:

$$
\left\{\begin{array}{l}
\text { Find } u^{0} \in V_{a} \text { such that } \forall v \in V_{a} \\
a\left(u^{0}, v\right)=\langle f, v\rangle_{V_{a}^{\prime} V_{a}} \text { for a given } f \in V_{a}^{\prime}
\end{array}\right.
$$

which is obviously continuous and coercive on $V_{a}$. It should be noticed that the hypothesis $f \in V_{a}^{\prime}$ is somewhat restrictive as we shall see later. When this hypothesis is satisfied the existence and uniqueness of $u^{0}$ in $V_{a}$ are ensured.

\section{The Problem $P(0)$}

\subsection{First considerations on the converge process}

In order to write down the equations and boundary conditions associated with classical integrations by parts, the space $L^{2}(\Omega)$ is identified with its dual; this will be used in the explicit description of dual spaces.

As follows from the completion process, $V$ is densely contained in $V_{a}$ so that

$$
V_{a}^{\prime} \subset V^{\prime} .
$$

Classical theory (Theorem 2.1) of convergence only holds for $f \in V_{a}^{\prime}$. In the case when $f \in V$, but $f \notin V_{a}^{\prime}$ general results of convergence are not available. The solution $u^{0}$ of the limit problem may or not exist but likely not in the finite energy space $V_{a}$. We recall that the energy of the solutions is defined by

$$
\begin{aligned}
& E\left(u^{\varepsilon}\right)=\frac{1}{2}\left[a\left(u^{\varepsilon}, u^{\varepsilon}\right)+\varepsilon^{2} b\left(u^{\varepsilon}, u^{\varepsilon}\right)\right] \\
& E\left(u^{0}\right)=\frac{1}{2} a\left(u^{0}, u^{0}\right) .
\end{aligned}
$$

The classical result is:

Theorem 2.1. Let $f \in V_{a}^{\prime}$ be fixed independently of $\varepsilon$. Let $u^{\varepsilon}$ and $u^{0}$ be the solutions of (1.5) and (1.11) respectively. Then

$$
u^{\varepsilon} \rightarrow u^{0} \text { in } V \text { strongly }
$$

and there exists a constant $C$ such that

$$
E\left(u^{\varepsilon}\right) \leq C
$$


The proof is classical, see for instance Section VI.1.3 in [18]. Let us recall that (2.5) follows from (1.5) with $v=u^{\varepsilon}$. Moreover, we have:

Theorem 2.2. Let $f \in V^{\prime}$ be fixed independently of $\varepsilon$. Let $u^{\varepsilon}$ the solution of (1.5), then

1. The necessary and sufficient condition for $E\left(u^{\varepsilon}\right)$ to remain bounded for $\varepsilon \searrow 0$ is that $f \in V_{a}^{\prime}$.

2. If $f \notin V_{a}^{\prime}$, then $E\left(u^{\varepsilon}\right)$ tends to infinity as $\varepsilon \searrow 0$.

Proof. In order to prove the first assertion, from Theorem 2.1, it is sufficient to prove that (2.5) implies $f \in V_{a}^{\prime}$. To this end, (2.5) implies that for a subsequence

$$
b\left(u^{\varepsilon}, u^{\varepsilon}\right) \leq \frac{C}{\varepsilon^{2}}
$$

and

$$
u^{\varepsilon} \rightarrow u^{*} \text { weakly in } V_{a}
$$

for some $u^{*} \in V_{a}$. Let us fix $v \in V$ in (1.5), then

$$
\begin{gathered}
a\left(u^{\varepsilon}, v\right) \rightarrow a\left(u^{*}, v\right) \\
\varepsilon^{2} b\left(u^{\varepsilon}, v\right) \leq \varepsilon^{2} b\left(u^{\varepsilon}, u^{\varepsilon}\right)^{\frac{1}{2}} b(v, v)^{\frac{1}{2}} \leq \varepsilon C \rightarrow 0
\end{gathered}
$$

so that

$$
a\left(u^{*}, v\right)=\langle f, v\rangle \forall v \in V .
$$

The left-hand side is a functional of the variable $v$ defined on $V$, continuous on $V_{a}$, so that the right-hand side is also, and this implies that $f \in V_{a}^{\prime}$.

To prove the second assertion, let us suppose that it is false. Then for a subsequence we should have (2.5) and in this case the proof of the first assertion shows that $f \in V_{a}^{\prime}$ what is a contradiction: Theorem 2.2 is proved.

Remark 1. We shall see that the space $V_{a}$ is somewhat "large" so that $V_{a}^{\prime}$ is "small" and $f \in V_{a}^{\prime}$ implies strong restrictions which are not satisfied by "usual" loadings $f$. Consequently, we are often in the situation of the second assertion of Theorem 2.2. This situation is in some sense "pathological". As we noted above, when $f \notin V_{a}^{\prime}$ the limit problem $P(0)$ may or not have a solution $u^{0}$ but if it does, it is not in the finite energy space $V_{a}$. This is the reason why we shall consider in the sequel solutions of the limit problem which are not variational solutions in $V_{a}$. An example of this situation is shown in [11] where it is seen that the energy confined in boundary and internal layers is not bounded (for $\varepsilon \searrow 0$ ) whereas the energy in outer regions does. Later on we shall search for the asymptotic behavior of $u^{\varepsilon}$ using the method of matched asymptotic expansions [20] which shall exhibit such kind of layers.

\subsection{A criterion for $f \in V_{a}^{\prime}$ and examples}

In this section, we shall consider functionals $f$ defined in (1.8) by functions $f_{i}$ (on $\Omega$ ) with $F_{i}=\mathcal{C}=0$. The form $a$ defined in (1.3) is associated with the two quantities

$$
\left\{\begin{array}{l}
\gamma_{1}(u) \equiv \partial_{1} u_{1} \\
\gamma_{2}(u) \equiv \partial_{2} u_{1}-u_{2} .
\end{array}\right.
$$


Let us define the injective application $\gamma$ by $(2.8)$

$$
V \stackrel{\gamma}{\rightarrow}\left(L^{2}\right)^{2}
$$

this application may be continued by continuity to $V_{a}$ to an isomorphism from $V_{a}$ onto its range $X$ which is clearly a closed subspace of $\left(L^{2}\right)^{2}$.

Let us now consider the functional

$$
l_{f}(v)=\int_{\Omega}\left(f_{1} v_{1}+f_{2} v_{2}\right) \mathrm{d} x
$$

defined on $V$. Then we have:

Theorem 2.3. The functional defined by (2.9) may be extended by continuity to $V_{a}$ (that is equivalent to $f \in V_{a}^{\prime}$ ) if, and only if, there exists $T=\left(T^{1}, T^{2}\right) \in\left(L^{2}\right)^{2}$ such that

$$
\int_{\Omega}\left(f_{1} v_{1}+f_{2} v_{2}\right) \mathrm{d} x=\left(T^{1}, \gamma_{1}(v)\right)_{L^{2}}+\left(T^{2}, \gamma_{2}(v)\right)_{L^{2}} \forall v \in V
$$

Proof. Let us suppose that there exists $T \in\left(L^{2}\right)^{2}$ which satisfies to (2.10), then the right-hand side in (2.10) is continuous on $V$ for the topology of $V_{a}$ and consequently the left hand side also does: this proves that the condition is sufficient. If (2.9) may be extended by continuity to $V_{a}$, then let us denote by

$$
\langle f, v\rangle_{V_{a}^{\prime} V_{a}}
$$

the functional so extended. Let $F$ be its image by the isomorphism $\gamma$, it is continuous on $X$ and defined by

$$
F(\xi)=\left\langle f, \gamma^{-1}(\xi)\right\rangle \forall \xi \in X
$$

From the Hahn-Banach theorem, we know that it may be extended to a functional $\tilde{F}(\xi)$ continuous on $\left(L^{2}\right)^{2}$ and, by the Riesz theorem, it may be expressed as the scalar product

$$
\tilde{F}(\xi)=\langle T, \xi\rangle_{\left(L^{2}\right)^{2}} \forall \xi \in\left(L^{2}\right)^{2}
$$

where $T$ is some element of $\left(L^{2}\right)^{2}$. In particular (2.13) holds for any $\xi \in X$ and even for any $\xi$ of the form $\xi=\gamma(v)$ with $v \in V$ (not necessarily to $V_{a}$ ). Then, from (2.13), we get

$$
\langle f, v\rangle \equiv \int_{\Omega}\left(f_{1} v_{1}+f_{2} v_{2}\right) \mathrm{d} x=(T, \gamma(v))_{\left(L^{2}(\Omega)\right)^{2}} \quad \forall v \in V
$$

the necessity of the condition is then proved.

Example 2.1. Let us consider the case when the whole boundary is clamped and let $f_{1}, f_{2}$ be smooth functions. We shall see that $f \in V_{a}^{\prime}$. Indeed, (2.10) explicitly writes

$$
\int_{\Omega}\left(f_{1} v_{1}+f_{2} v_{2}\right) \mathrm{d} x=\int_{\Omega}\left[T_{1} \partial_{1} v_{1}+T_{2}\left(\partial_{2} v_{1}-v_{2}\right)\right] \mathrm{d} x \quad \forall v \in V
$$


Taking $v \in[\mathcal{D}(\Omega)]^{2}$ we obtain

$$
\left\{\begin{array}{l}
f_{1}=-\partial_{1} T_{1}-\partial_{2} T_{2} \\
f_{2}=-T_{2}
\end{array}\right.
$$

As the two components $f_{1}$ and $f_{2}$ are smooth it is possible to construct smooth $T^{1}$ and $T^{2}$, in particular $\left(T^{1}, T^{2}\right) \in\left(L^{2}\right)^{2}$, satisfying $(2.15)$. Then we have

$$
\int_{\Omega}\left(f_{1} v_{1}+f_{2} v_{2}\right) \mathrm{d} x=\int_{\Omega}\left[\left(-\partial_{1} T_{1}-\partial_{2} T_{2}\right) v_{1}+\left(-T_{2}\right) v_{2}\right] \mathrm{d} x \quad \forall v \in V
$$

In the considered case of a clamped boundary we have $V=H_{0}^{1} \times H_{0}^{2}$ so that an obvious integration by parts in (2.16) gives (2.14). It then follows from Theorem 2.3 that

$$
f \in V_{a}^{\prime}
$$

Consequently, from Theorem 2.2 we see that the solution $u^{\varepsilon}$ of $(2.12)$ is such that the energy $E\left(u^{\varepsilon}\right)$ of $u^{\varepsilon}$ remains bounded as $\varepsilon \searrow 0$.

Example 2.2. We consider again a totaly clamped boundary but $f_{i}$ are no more smooth functions. We shall take

$$
\left\{\begin{array}{l}
f_{1} \equiv 0 \\
f_{2}=\left\{\begin{array}{l}
0 \text { if } x_{2}<\pi / 2 \\
1 \text { if } x_{2}>\pi / 2
\end{array}\right.
\end{array}\right.
$$

the given forces defined on the domain $\Omega$. We emphasize that the discontinuity of $f$ is along a curve $x_{2}=$ const. We shall see later that these curves are the characteristics of the problem $P(0)$. Let us prove that $f \notin V_{a}^{\prime}$.

Indeed, we are showing that $T_{1}, T_{2} \in L^{2}$ and satisfying (2.14) cannot exist. If this was the case then taking $v \in[\mathcal{D}(\Omega)]^{2}$ we should have (2.15). In addition, as $f_{1}, f_{2}$ are piecewise smooth the corresponding traces of $T_{1} n_{1}+T_{2} n_{2}$ make sense, allowing classical integration by parts. Then, from (2.14) with arbitrary $v$ we get

$$
\left[\left|T_{2}\right|\right]=0 \text { on } x_{2}=\frac{\pi}{2}
$$

where the brackets denote the jump across the discontinuity. We then see that (2.19) with (2.15) and (2.18) are not compatible. So that $T_{1}, T_{2}$ cannot exist. According to Theorem 2.3 the given force $f$ does not belong to $V_{a}^{\prime}$. Consequently, from Theorem 2.2 we see that the solution $u^{\varepsilon}$ of $(2.12)$ is such that the energy $E\left(u^{\varepsilon}\right)$ tends to infinity as $\varepsilon \searrow 0$.

Example 2.3. Now we consider a smooth given force $f$ but the boundary is constituted of two parts $\Gamma_{0}$ and $\Gamma_{1}$. We recall that $\Gamma_{0}$ is clamped while $\Gamma_{1}$ is free. We shall take as $\Gamma_{1}$ the part $x_{2}=0$ of the boundary. We emphasize that, as in the previous example, this free boundary is along a characteristic of the problem $P(0)$. The space $V$ is then defined as

$$
V=\left\{v_{1} \in H^{1}(\Omega) ;\left.v\right|_{\Gamma_{0}=0}\right\} \times\left\{v_{2} \in H^{2}(\Omega) ;\left.v\right|_{\Gamma_{0}}=\left.\frac{\partial v}{\partial n}\right|_{\Gamma_{0}}=0\right\}
$$


Exactly as in the previous example if $T_{1}, T_{2}$ exist, then they satisfy

$$
\left\{\begin{array}{l}
\left\{\begin{array}{l}
-\partial_{1} T_{1}-\partial_{2} T_{2}=f_{1} \\
T_{2}=f_{2}
\end{array}\right. \\
T_{2}=0 \text { on } \Gamma_{1} .
\end{array}\right.
$$

It is clear that $(2.21)_{2}$ and $(2.21)_{3}$ are compatible if and only if $\left.f_{2}\right|_{\Gamma_{1}}=0$. Oppositely, when this condition is satisfied we may construct smooth functions $T_{1}, T_{2}$ satisfying $(2.21)$ and classical integration by parts show that $T_{1}, T_{2}$ satisfy (2.10). Then $f_{2}=0$ on $\Gamma_{1}$ is the necessary and sufficient condition for $f \in V_{a}^{\prime}$. Obviously it is also the necessary and sufficient condition for $E\left(u^{\varepsilon}\right)$ to remain bounded as $\varepsilon \searrow 0$.

\subsection{Equations and boundary conditions of $P(0)$}

The variational formulation (1.11) of the limit problem is somewhat abstract as $V_{a}$ was only defined by completion. We have not a precise description of $V_{a}$ which depends on the disposition of $\Gamma_{0}$ and $\Gamma_{1}$. But in any case it follows from (1.10) and (1.3) that $v \in V_{a} \Rightarrow \partial_{1} v_{1} \in L^{2}(\Omega)$. Moreover, from the fact that $\Gamma_{0}$ contains at least one of the vertical segments of the boundary and Poincaré inequality for $x_{2}=$ const., it follows that $v_{1} \in L^{2}(\Omega)$. Consequently, the principal boundary condition $v_{1}=0$ is inherited by $V_{a}$ on the parts of $\Gamma_{0}$ which are tranversal to $x_{2}=$ const.

As for $v_{2}$, if $v \in V_{a}$, it follows from (1.10) and (1.3) that $v_{2}$ is the sum of an element of $L^{2}(\Omega)$ and $\partial_{2} v_{1}$ so that the principal boundary conditions of $v_{1}$ on $x_{2}=$ const. and of $v_{2}$ everywhere are lost in the completion process. This allows us to write down the equations and boundary conditions of $P(0)$ (without a precise description of $\left.V_{a}\right)$ :

$$
\begin{gathered}
\left\{\begin{array}{l}
-\Delta u_{1}+\partial_{2} u_{2}=f_{1} \\
-\partial_{2} u_{1}+u_{2}=f_{2}
\end{array}\right. \\
\left\{\begin{array}{l}
u_{1}=0 \text { on the parts of } \Gamma_{0} \text { transversal to } x_{2}=\text { const. } \\
\partial_{1} u_{1}=0 \text { on the parts of } \Gamma_{1} \text { transversal to } x_{2}=\text { const. }
\end{array}\right.
\end{gathered}
$$

obviously, the first of these boundary conditions is a principal one on $V_{a}$ as we said above, whereas the second one is a natural condition coming from integration by parts.

Clearly (2.22) is equivalent to

$$
\begin{aligned}
-\partial_{1}^{2} u_{1} & =f_{1}-\partial_{2} f_{2} \\
u_{2} & =f_{2}+\partial_{2} u_{1} .
\end{aligned}
$$

Under this form it is obvious that the limit problem is essentially equivalent to $(2.24)$ for $u_{1}$. This equation is an elliptic one with respect to $x_{1}$ with parameter $x_{2}$. In $\Omega$ it is parabolic with double characteristics $x_{2}=$ const. As $x_{2}$ appears as a parameter, $f_{1}$ and $f_{2}$ may be chosen to be distributions of $x_{2}$ with values in an appropriated space for the variable $x_{1}$. Consequently, when $f_{2}$ is not sufficiently smooth with respect to $x_{2}$, the equations (2.22) and the boundary conditions (2.23) keep a sense in a more general framework which is not that of the variational problem (1.11).

Let us define the operator $A$ by

$$
A u=-\partial_{1}^{2} u_{1}
$$

for functions depending on the variable $x_{1}$ and the evident boundary conditions coming from (2.23). We note that according to the hypothesis that $\Gamma_{0}$ and $\Gamma_{1}$ are made of whole sides of the boundary of $\Omega$, this operator is 
independent of $x_{2}$ so that it commutes with differentiations with respect this parameter. Obviously, the solution of (2.24), (2.25) then writes

$$
\left\{\begin{array}{l}
u_{1}\left(\bullet, x_{2}\right)=A^{-1}\left[f_{1}\left(\bullet, x_{2}\right)-\partial_{2} f_{2}\left(\bullet, x_{2}\right)\right] \\
u_{2}\left(\bullet, x_{2}\right)=f_{2}\left(\bullet, x_{2}\right)+A^{-1}\left[\partial_{2} f_{1}\left(\bullet, x_{2}\right)-\partial_{2}^{2} f_{2}\left(\bullet, x_{2}\right)\right] .
\end{array}\right.
$$

\subsection{Solutions in the sense of distributions}

According to the previous considerations, we now consider the system (2.22) or (2.24) and (2.25) as well as the solution (2.27) in the sense of distributions of $x_{2}$ with values in a space (for instance $L^{2}(0, \pi)$ ) of the variable $x_{1}$. In order to exhibit the singular terms, we consider the case when $f_{1}=0$ and $f_{2}$ is a piecewise smooth function of the variable $x_{2}$ with values in $L^{2}(0, \pi)$ with a discontinuity at a point $\gamma$ of $(0, \pi)$. Let $\varphi$ and $\psi \in L^{2}(0, \pi)$ be the jumps of the function and the first derivative at $x_{2}=\gamma$. The solution $(2.27)$ takes the form

$$
\left\{\begin{array}{l}
u_{1}\left(\bullet, x_{2}\right)=-A^{-1}\left(\partial_{2}^{f} f_{2}\right)-\left(A^{-1} \varphi(\bullet)\right) \delta_{\gamma} \\
u_{2}\left(\bullet, x_{2}\right)=f_{2}\left(\bullet, x_{2}\right)-A^{-1}\left(\partial_{2}^{f 2} f_{2}\left(\bullet, x_{2}\right)\right)-\left(A^{-1} \psi(\bullet)\right) \delta_{\gamma}-\left(A^{-1} \varphi(\bullet)\right) \delta_{\gamma}^{\prime}
\end{array}\right.
$$

where $\partial_{2}^{f}$ denotes the derivative in the sense of functions. To fix ideas, let us take

$$
\left\{\begin{array}{l}
f_{1}=0 \\
f_{2}\left(\bullet, x_{2}\right)= \begin{cases}0 & \text { for } x_{2}<\gamma \\
\varphi(\bullet) & \text { for } x_{2}>\gamma\end{cases}
\end{array}\right.
$$

in this case the solution is

$$
\left\{\begin{array}{l}
u_{1}\left(\bullet, x_{2}\right)=-\left(A^{-1} \varphi(\bullet)\right) \delta_{\gamma} \\
u_{2}\left(\bullet, x_{2}\right)=f_{2}\left(\bullet, x_{2}\right)-\left(A^{-1} \varphi(\bullet)\right) \delta_{\gamma}^{\prime}
\end{array}\right.
$$

We shall see later that these solutions of problem $P(0)$ are the limits of the corresponding solutions of problem $P(\varepsilon)$ as $\varepsilon \searrow 0$. Clearly this implies that the solutions of $P(\varepsilon)$ involve boundary (in fact internal) layers terms which converge to the singular terms of (2.30).

\subsection{Localization of the boundary and internal layers}

As a consequence of Section 2.4 it appears that the problem $P(\varepsilon)$ exhibits internal layers terms along the segments $x_{2}=$ const. where $f$ is not smooth as function of $x_{2}$ with values in $L^{2}(0, \pi)$ for instance. The structure of the internal layer depends highly on the degree of non-smoothness of $f$. We emphazise that $x_{2}=$ const. are the characteristics of the limit problem (2.24).

The explicit solution (2.30) exhibits an example of propagation of singularities along the characteristics. As the operator $A^{-1}$ of the variable $x_{1}$ is non-local, we may have $\varphi$ with compact support in $(0, \pi)$ whereas the solution $u$ is singular along the whole segment of characteristic $x_{2}=$ const., $x_{1} \in(0, \pi)$.

Oppositely, let us consider the case when $f_{1}=0$ and $f_{2}$ is piecewise constant with a discontinuity along a curve $\mathcal{C}$ which is transversal to the characteristics. The method of solution with parameter $x_{2}$ shows that $u$ is smooth unless on $\mathcal{C}$ where the function $u_{2}$ and the first derivative of $u_{1}$ have jumps. Clearly, in this case, $u^{\varepsilon}$ exhibits an internal layer along the curve $\mathcal{C}$. In addition, if one of the extremities of $\mathcal{C}$ is a point interior to $\Omega$, the solution is singular at this point and propagates from it along $x_{2}=$ const.

On the other hand, as $x_{2}$ is a parameter, the limit problem $P(0)$ has no boundary condition on $x_{2}=0$ and $x_{2}=1$ so that $u^{\varepsilon}$ exhibits boundary layers along these (characteristic) boundaries.

Also boundary layers appear along the parts of $\Gamma_{0}$ transversal to the characteristics (vertical parts of $\Gamma_{0}$ ) as the boundary conditions (1.1) involving $u_{2}$ disappear in the limit (compare with (2.23)). We shall call them non-characteristic boundary layers. 


\section{Scaling in the layers. Method of exponential solutions}

Classically [20], in order to describe the structure of a layer we must perform a change of variables including a dilatation of the variable normal to the layer. Moreover, in order to obtain a consistent system, a rescaling of the unknowns is usually needed. The deduction by a classical procedure of the appropriate scalings is possible but we use here a method issued from the analysis of the structure of the exponential solutions of the homogeneous system [15]. It should be pointed out that this method presents analogies with the study of asymptotic solutions of systems using the eikonal equation ([18], Sect. III.4).

\subsection{Characteristic layers}

We first consider the case of the layers along $x_{2}=$ const. As the characteristics of the limit problem are normal to the vector $(0,1)$ there exist solutions of the form

$$
u\left(x_{1}, x_{2}\right)=v e^{i\left(\xi_{1} x_{1}+\xi_{2} x_{2}\right)}
$$

with $\xi_{1}=0, \xi_{2} \neq 0$. Let us search, for $\varepsilon>0$, solutions of the form

$$
u^{\varepsilon}\left(x_{1}, x_{2}\right)=v^{\varepsilon} e^{i \xi_{1}+\mu x_{2}}
$$

with $|\mu| \rightarrow+\infty$ (i.e. such that $\left(\xi_{1}(\right.$ real $\left.), \xi_{2} \equiv-i \mu\right)$ tends to be proportional to $\left.(0,1)\right)$. The solutions $(3.1)$ are sinusoidal in $x_{1}$ with wave length of order $\mathcal{O}(1)$ and very fast variations in $x_{2}$. By substitution of (3.1) in the homogeneous system associated with (1.6) we obtain

$$
\left\{\begin{array}{l}
\left(\xi_{1}^{2}-\mu^{2}\right) v_{1}^{\varepsilon}+\mu v_{2}^{\varepsilon}=0 \\
-\mu v_{1}^{\varepsilon}+v_{2}^{\varepsilon}+\varepsilon^{2}\left[\left(\xi_{1}^{2}-\mu^{2}\right)^{2} v_{2}^{\varepsilon}+\left(\xi_{1}^{2}-\mu^{2}\right) v_{2}^{\varepsilon}+v_{2}^{\varepsilon}\right]=0 .
\end{array}\right.
$$

The vanishing of the determinant of system (3.2) gives

$$
\xi_{1}^{2}+\varepsilon^{2}\left(\xi_{1}^{2}-\mu^{2}\right)\left[\left(\xi_{1}^{2}-\mu^{2}\right)^{2}+\xi_{1}^{2}-\mu^{2}+1\right]=0
$$

which for finite $\xi_{1}$ and $\mu \rightarrow+\infty$ gives the behavior of $\mu$ :

$$
\mu \cong e^{\frac{i k \pi}{3}} \xi_{1}^{\frac{1}{3}} \varepsilon^{-\frac{1}{3}}
$$

Then, from the first equation (3.2) we see that

$$
\frac{v_{2}^{\varepsilon}}{v_{1}^{\varepsilon}}=\mathcal{O}\left(\varepsilon^{-\frac{1}{3}}\right)
$$

It then appears that the just obtained solutions with $\xi_{1}=\mathcal{O}(1), \mu=\mathcal{O}\left(\varepsilon^{-\frac{1}{3}}\right)$ have a characteristic length of variation in the $x_{2}$ direction of order $\mathcal{O}\left(\varepsilon^{\frac{1}{3}}\right)$; this corresponds to a layer of thickness $\mathcal{O}(\eta)$,

$$
\eta=\varepsilon^{\frac{1}{3}}
$$

Moreover, the corresponding scaling of $u$ is

$$
\frac{u_{2}^{\varepsilon}}{u_{1}^{\varepsilon}}=\mathcal{O}\left(\eta^{-1}\right)
$$




\subsection{Non-characteristic layers}

Analogously, in the case of layers parallel to $x_{1}=$ const. we search solutions of the form

$$
u^{\varepsilon}\left(x_{1}, x_{2}\right)=v^{\varepsilon} e^{\mu x_{1}+i \xi_{2} x_{2}}
$$

from which it follows that

$$
\mu \cong(-1)^{\frac{1}{4}} \varepsilon^{-\frac{1}{2}}
$$

so that the thickness of the layer is of order

$$
\eta=\varepsilon^{\frac{1}{2}}
$$

and the scaling of $u$ is

$$
\frac{u_{2}^{\varepsilon}}{u_{1}^{\varepsilon}}=\mathcal{O}\left(\eta^{-1}\right)=\mathcal{O}\left(\varepsilon^{-\frac{1}{2}}\right)
$$

\section{Boundary LAYER ALONG A CHARACTERIStiC PART OF $\Gamma_{0}$}

As announced at the end of Section 2.5, there exists a boundary layers along the boundaries $x_{2}=0$ and $x_{2}=\pi$. Their structure is different according to the boundary belongs to $\Gamma_{0}$ or to $\Gamma_{1}$. In this section we are concerned by the first case and we suppose that the boundary is $x_{2}=0$. We also suppose, to fix ideas that $\Gamma_{0}$ contains the vertical segments $x_{1}=0$ and $x_{1}=\pi$. According to Section 3.1, the appropriate scaling is then (3.5) and (3.6) so we define the dilatation by

$$
y_{2}=\frac{x_{2}}{\eta}
$$

and we search for asymptotic expansions of the form

$$
\left\{\begin{array}{l}
u_{1}^{\varepsilon}\left(x_{1}, x_{2}\right)=U_{1}^{\eta}\left(x_{1}, y_{2}\right)=U_{1}^{0}\left(x_{1}, y_{2}\right)+\cdots \\
u_{2}^{\varepsilon}=\eta^{-1} U_{2}^{\eta}\left(x_{1}, y_{2}\right)=\eta^{-1} U_{2}^{0}\left(x_{1}, y_{2}\right)+\cdots
\end{array}\right.
$$

Tentatively, we took the component $u_{1}$ of order $\mathcal{O}(1)$ in order to match it with the outer expansion (we recall that only the ratio $u_{2} / u_{1}$ was defined by (3.6)). In the sequel, we shall denote by $D_{2}$ the derivative with respect to $y_{2}$ :

$$
D_{2}=\eta \partial_{2}
$$

According to the boundary layer theory [20], the expansions (4.2) holds true in the domain $D$ defined by $x_{1} \in(0, \pi), y_{2} \in(0,+\infty)$. The solution $U^{\eta}$ must satisfy the boundary conditions

$$
\left\{\begin{array}{l}
U_{1}^{\eta}\left(x_{1}, 0\right)=U_{2}^{\eta}\left(x_{1}, 0\right)=D_{2} U_{2}^{\eta}\left(x_{1}, 0\right)=0 \\
U_{1}^{\eta}\left(0, y_{2}\right)=U_{1}^{\eta}\left(\pi, y_{2}\right)=0
\end{array}\right.
$$

and the matching conditions for $y_{2} \rightarrow+\infty$ which shall consider later. 
The variational formulation of (1.5) must hold true in the domain $D$ at least for test functions with bounded support in $y_{2}$, we shall take them depending on $\eta$ in a form analogous to (4.2). Consequently we have

$$
\begin{gathered}
\int_{D}\left\{\partial_{1} U_{1}^{\eta} \partial_{1} V_{1}^{\eta}+\frac{1}{\eta^{2}}\left(D_{2} U_{1}^{\eta}-U_{2}^{\eta}\right)\left(D_{2} V_{1}^{\eta}-V_{2}^{\eta}\right)+\frac{\varepsilon^{2}}{\eta^{6}}\left[D_{2}^{2} U_{2}^{\eta} D_{2}^{2} V_{2}^{\eta}+\cdots\right]\right\} \mathrm{d} x_{1} \mathrm{~d} y_{2} \\
=\int_{D}\left\{\left[f_{1}\left(x_{1}, 0\right)+\cdots\right] V_{1}^{\eta}+\frac{1}{\eta}\left[f_{2}\left(x_{1}, 0\right)+\eta y_{2} \partial_{2} f_{2}\left(x_{1}, 0\right)+\cdots\right] V_{2}^{\eta}\right\} \mathrm{d} x_{1} \mathrm{~d} y_{2}
\end{gathered}
$$

where $f$ is supposed to be regular and was represented by its Taylor expansion in the vicinity of $x_{2}=0$. We note that, according to $(3.5), \varepsilon^{2} / \eta^{6}=1$ in formula (4.5). Taking $V_{1}^{0}=0$ and $V_{2}^{0} \in \mathcal{D}(D)$, the leading term (of order $\eta^{-2}$ ) gives

$$
D_{2} U_{1}^{0}-U_{2}^{0}=0
$$

which implies a constraint for the unknown $U^{0}$. In the sequel, we shall take test functions satisfying this constraint, thus the test functions will satisfy

$$
V_{2}^{0}=D_{2} V_{1}^{0}
$$

Consequently, the term of order $1 / \eta$ in $(4.5)$ vanishes, indeed it is

$$
\int_{D} f_{2}\left(x_{1}, 0\right) V_{2}^{0}\left(x_{1}, y 2\right) d \mathrm{x}_{1} \mathrm{~d} y_{2}=\int_{0}^{\pi} f_{2}\left(x_{1}, 0\right)\left(\int_{0}^{+\infty} D_{2} V_{1}^{0}\left(x_{1}, y_{2}\right) \mathrm{d} y_{2}\right) \mathrm{d} x_{1}=0
$$

as $V_{1}^{0}$ vanishes for $y_{2}=0$ and $y_{2}$ sufficiently large.

Presently the leading term of (4.5) is of order $\mathcal{O}(1)$ and must vanish so that we have

$$
\int_{D}\left[\partial_{1} U_{1}^{0} \partial_{1} V_{1}^{0}+D_{2}^{2} U_{2}^{0} D_{2}^{2} V_{2}^{0}\right] \mathrm{d} x_{1} \mathrm{~d} y_{2}=\int_{D}\left[f_{1}\left(x_{1}, 0\right) V_{1}^{0}+y_{2} \partial_{2} f_{2}\left(x_{1}, 0\right) V_{2}^{0}\right] \mathrm{d} x_{1} \mathrm{~d} y_{2}
$$

Taking account of (4.6) and (4.7), the problem reduces to another one for the only unknown $U_{1}^{0}$ (with the only test function $V_{1}^{0}$ ) which, after an integration by parts in the right-hand side, writes

$$
\int_{D}\left[\partial_{1} U_{1}^{0} \partial_{1} V_{1}^{0}+D_{2}^{3} U_{1}^{0} D_{2}^{3} V_{1}^{0}\right] \mathrm{d} x_{1} \mathrm{~d} y_{2}=\int_{D}\left[f_{1}\left(x_{1}, 0\right)-\partial_{2} f_{2}\left(x_{1}, 0\right)\right] V_{1}^{0} \mathrm{~d} x_{1} \mathrm{~d} y_{2}
$$

from which, taking $V_{1}^{0} \in \mathcal{D}(\Omega)$, we obtain the equation satisfied by $U_{1}^{0}$ :

$$
-\partial_{1}^{2} U_{1}^{0}-D_{2}^{6} U_{1}^{0}=f_{1}\left(x_{1}, 0\right)-\partial_{2} f_{2}\left(x_{1}, 0\right) .
$$

According to (4.4) and taking account of (4.6), the component $U_{1}^{0}$ must satisfy the boundary conditions

$$
U_{1}^{0}\left(x_{1}, 0\right)=D_{2} U_{1}^{0}\left(x_{1}, 0\right)=D_{2}^{2} U_{1}^{0}\left(x_{1}, 0\right)=0
$$

and

$$
U_{1}^{0}\left(0, y_{2}\right)=U_{1}^{0}\left(\pi, y_{2}\right)=0
$$


The solution of (4.9) may be written under the form

$$
U_{1}^{0}\left(x_{1}, y_{2}\right)=\hat{U}_{1}^{0}\left(x_{1}\right)+\tilde{U}_{1}^{0}\left(x_{1}, y_{2}\right)
$$

where $\hat{U}_{1}^{0}\left(x_{1}, 0\right)$ is the solution of

$$
-\hat{U}_{1}^{0 \prime}\left(x_{1}\right)=f_{1}\left(x_{1}, 0\right)-\partial_{2} f_{2}\left(x_{1}, 0\right) \equiv F\left(x_{1}\right)
$$

with the boundary conditions

$$
\hat{U}_{1}^{0}(0)=\hat{U}_{1}^{0}(\pi)=0
$$

i.e. of equation (2.24) and the boundary conditions $(2.23)$ so that $\hat{U}_{1}^{0}$ is nothing but $u_{1}\left(x_{1}, 0\right)$ in Section 2.3 .

As for the new unknown $\tilde{U}_{1}^{0}\left(x_{1}, y_{2}\right)$ it is solution of the homogeneous equation

$$
-\partial_{1}^{2} \tilde{U}_{1}^{0}-D_{2}^{6} \tilde{U}_{1}^{0}=0 .
$$

Let us search a solution of (4.15) under the form

$$
\tilde{U}_{1}^{0}\left(x_{1}, y_{2}\right)=\sum_{n=1}^{+\infty} a_{n}\left(y_{2}\right) \sin n x_{1}
$$

we have

$$
\tilde{U}_{1}^{0}\left(x_{1}, y_{2}\right)=\sum_{n=1}^{+\infty} a_{n}\left(y_{2}\right) \sin n x_{1}=U_{1}^{0}\left(x_{1}, y_{2}\right)-\hat{U}_{1}^{0}\left(x_{1}\right) .
$$

Now, let us write the Fourier expansion of $F\left(x_{1}\right)$ under the form

$$
F\left(x_{1}\right)=\sum_{n=1}^{+\infty} F_{n} \sin n x_{1}
$$

then, from the conditions (4.10), the coefficients $a_{n}$ must satisfy

$$
\begin{aligned}
& a_{n}(0)=-\frac{F_{n}}{n^{2}} \\
& a_{n}^{\prime}(0)=a_{n}^{\prime \prime}(0)=0
\end{aligned}
$$

and, from (4.15),

$$
n^{2} a_{n}\left(y_{2}\right)-\frac{\mathrm{d}^{6}}{\mathrm{~d} y_{2}^{6}} a_{n}\left(y_{2}\right)=0 .
$$

The bounded solution of (4.19) is of the form

$$
a_{n}\left(y_{2}\right)=\sum_{k=2}^{4} b_{k} \exp \left\{n^{\frac{1}{3}} \exp \left(\frac{i k \pi}{3}\right) y_{2}\right\}
$$


where the coefficients are uniquely determined on account of (4.17) and (4.18). Moreover, as $y_{2} \rightarrow+\infty$, $U_{1}^{0}\left(x_{1}, y_{2}\right)$ tends to $\hat{U}_{1}^{0}\left(x_{1}\right)$ solution of the limit problem $P(0)$ that insures the matching with the solution of the limit problem $P(0)$ of Section 2.3.

As for the component $U_{2}^{0}$, as we saw it is given by

$$
U_{2}{ }^{0}\left(x_{1}, y_{2}\right)=D_{2} U_{1}{ }^{0}\left(x_{1}, y_{2}\right)=D_{2} \tilde{U}_{1}^{0}\left(x_{1}, y_{2}\right)
$$

and is such that

$$
U_{2}^{0}\left(x_{1}, y_{2}\right) \underset{y_{2} \rightarrow+\infty}{\longrightarrow} 0
$$

that constitutes the matching condition as $u_{2}^{\varepsilon}$ is of order $\varepsilon^{-\frac{1}{3}}$ in the layer and $\mathcal{O}(1)$ out of it.

\section{Boundary LAYER AlONG A CHARACTERISTiC PART OF $\Gamma_{1}$}

In this section we consider the case when $\Gamma_{1}$ contains the boundary $x_{2}=0$ and $\Gamma_{0}$ contains $x_{1}=0$ and $x_{1}=\pi$. We consider the layer along $x_{2}=0$. As in the previous section, the scaling of the $x_{2}$ is defined by (4.1) but the asymptotic expansions are searched under the form

$$
\left\{\begin{array}{l}
u_{1}^{\varepsilon}\left(x_{1}, x_{2}\right)=\theta(\eta) U_{1}^{\eta}\left(x_{1}, y_{2}\right)=\theta(\eta) U_{1}^{0}\left(x_{1}, y_{2}\right)+\cdots \\
u_{2}^{\varepsilon}=\eta^{-1} \theta(\eta) U_{2}^{\eta}\left(x_{1}, y_{2}\right)=\eta^{-1} \theta(\eta) U_{2}^{0}\left(x_{1}, y_{2}\right)+\cdots
\end{array}\right.
$$

where $\theta(\eta)$ is to be determined later. Indeed, the method introduced in Section 3 only gives the ratio $\frac{u_{2}}{u_{1}}(c . f$. (3.6)) that is satisfied by (5.1); we shall see later that different functions $\theta(\eta)$ should be chosen according to the behavior of $f_{2}$ on the free boundary. We shall again denote by $D_{2}$ the derivative with respect to $y_{2}$ which, of course, satisfies (4.3).

As before, the variational formulation (1.5) must hold true in the domain $D$ for test functions with compact support in $y_{2}$ and we shall choose them depending on $\eta$ in a form analogous to (5.1) then, we have

$$
\begin{gathered}
\theta(\eta) \int_{D}\left\{\partial_{1} U_{1}^{\eta} \partial_{1} V_{1}^{\eta}+\frac{1}{\eta^{2}}\left(D_{2} U_{1}^{\eta}-U_{2}^{\eta}\right)\left(D_{2} V_{1}^{\eta}-V_{2}^{\eta}\right)+\left[D_{2}^{2} U_{2}^{\eta} D_{2}^{2} V_{2}^{\eta}+\cdots\right]\right\} \mathrm{d} x_{1} \mathrm{~d} y_{2}= \\
\int_{D}\left\{\left[f_{1}\left(x_{1}, 0\right)+\cdots\right] V_{1}^{\eta}+\frac{1}{\eta}\left[f_{2}\left(x_{1}, 0\right)+\eta y_{2} \partial_{2} f_{2}\left(x_{1}, 0\right)+\cdots\right] V_{2}^{\eta}\right\} \mathrm{d} x_{1} \mathrm{~d} y_{2}
\end{gathered}
$$

Taking $V_{1}^{0}=0$ and arbitrary $V_{2}^{0}$, the leading term in the left-hand side is

$$
\frac{\theta(\eta)}{\eta^{2}} \int_{D}\left(D_{2} U_{1}^{0}-U_{2}^{0}\right) V_{2}^{0} \mathrm{~d} x_{1} \mathrm{~d} y_{2}
$$

and in the right-hand side is

$$
\frac{1}{\eta} \int_{D} f_{2}\left(x_{1}, 0\right) V_{2}^{0} \mathrm{~d} x_{1} \mathrm{~d} y_{2}
$$

consequently, it immediately appears, taking account of the matching condition, that we have only two possibilities: $\theta(\eta)>>1$ or $\theta(\eta)=1$. Then, as we shall see Sections 5.1 and 5.2 , in order to obtain problems consistent with the data, the function $\theta(\eta)$ must be chosen as follows, according to the value of the component $f_{2}$ at 
the boundary $x_{2}=0$

$$
\begin{array}{|l|l|}
\hline f_{2}\left(x_{1}, 0\right) \neq 0 & \theta(\eta)=\eta^{-1} \\
\hline f_{2}\left(x_{1}, 0\right)=0 & \theta(\eta)=1 \\
\hline
\end{array}
$$

with in any case $D_{2} U_{1}^{0}-U_{2}^{0}=0$ i.e. (4.6).

5.1. Case when $f_{2}\left(x_{1}, 0\right) \neq 0$

In this case, taking test functions such that $V_{2}^{0}=D_{2} V_{1}^{0}$ in the variational formulation (5.2), its leading term is

$$
\frac{1}{\eta} \int_{D}\left\{\partial_{1}^{2} U_{1}^{0} V_{1}^{0}+D_{2}^{3} U_{1}^{0} D_{2}^{3} V_{1}^{0}\right\} \mathrm{d} x_{1} \mathrm{~d} y_{2}=\int_{D} \frac{1}{\eta} f_{2}\left(x_{1}, 0\right) D_{2} V_{1}^{0} \mathrm{~d} x_{1} \mathrm{~d} y_{2}
$$

which, after evident integrations by parts, becomes

$$
\begin{aligned}
\int_{D}\left[-\partial_{1}^{2} U_{1}^{0}-\right. & \left.D_{2}^{6} U_{1}^{0}\right] V_{1}^{0} \mathrm{~d} x_{1} \mathrm{~d} y_{2}+\int_{\partial D}\left\{\left[\partial_{1} U_{1}^{0} V_{1}^{0}\right] n_{1}\right. \\
& \left.+\left[D_{2}^{3} U_{1}^{0} D_{2}^{2} V_{1}^{0}-D_{2}^{4} U_{1}^{0} D_{2} V_{1}^{0}+D_{2}^{5} U_{1}^{0} V_{1}^{0}\right] n_{2}\right\} \mathrm{d} s \\
& =\int_{\Gamma_{1}} f_{2}\left(x_{1}, 0\right) V_{1}^{0} n_{2} \mathrm{~d} x_{1} .
\end{aligned}
$$

Then, taking $V_{1}^{0} \in \mathcal{D}(D)$, we obtain the equation

$$
-\partial_{1}^{2} U_{1}^{0}-D_{2}^{6} U_{1}^{0}=0
$$

and, from the integrals along the boundary (as $D_{2}^{2} V_{1}^{0}, D_{2} V_{1}^{0}$ and $V_{1}^{0}$ are arbitrary on the part $x_{2}=0$ of $\partial D$ ), we have

$$
\left\{\begin{array}{l}
D_{2}^{3} U_{1}^{0}\left(x_{1}, 0\right)=0 \\
D_{2}^{4} U_{1}^{0}\left(x_{1}, 0\right)=0 \\
D_{2}^{5} U_{1}^{0}\left(x_{1}, 0\right)=f_{2}\left(x_{1}, 0\right)
\end{array}\right.
$$

As in Section 4, we now search for a solution of the form

$$
U_{1}^{0}\left(x_{1}, y_{2}\right)=\sum_{n=1}^{+\infty} a_{n}\left(y_{2}\right) \sin n x_{1}
$$

then the coefficients $a_{n}\left(y_{2}\right)$ are the bounded solution of the equation

$$
-a_{n}^{(6)}\left(y_{2}\right)+n^{2} a_{n}\left(y_{2}\right)=0
$$

i.e. of the form

$$
a_{n}\left(y_{2}\right)=\sum_{k=2}^{4} c_{k} \exp \left[n^{\frac{1}{3}} \exp \left(\frac{i k \pi}{3}\right) y_{2}\right]
$$


where the coefficients $c_{k}$ are determined by the boundary conditions

$$
\left\{\begin{array}{l}
a_{n}^{(3)}(0)=a_{n}^{(4)}(0)=0 \\
a_{n}^{(5)}(0)=F_{n}
\end{array}\right.
$$

where $F_{n}$ is the coefficient of $\sin n x_{1}$ in the Fourier expansion of $f_{2}\left(x_{1}, 0\right)$.

Of course, $U_{2}^{0}\left(x_{1}, y_{2}\right)$ is then given by (4.6).

\subsection{Case when $f_{2}\left(x_{1}, 0\right)=0$}

This case, for which $\theta(\eta)=1$, is worked out exactly as the previous one the only modifications concern the equation which becomes

$$
-\partial_{1}^{2} U_{1}^{0}-D_{2}^{(6)} U_{2}^{0}=f_{1}\left(x_{1}, 0\right)
$$

and the last boundary condition in (5.7) which is now

$$
D_{2}^{(5)} U_{1}^{0}\left(x_{1}, 0\right)=0 .
$$

Consequently, the analogous of the coefficients $a_{n}\left(y_{2}\right)$ are solutions of

$$
-a_{n}^{(6)}\left(y_{2}\right)+n^{2} a_{n}\left(y_{2}\right)=f_{1}\left(x_{1}, 0\right)
$$

with the boundary conditions

$$
a_{n}^{(3)}(0)=a_{n}^{(4)}(0)=a_{n}^{(5)}(0)=0 .
$$

It should be easily seen that the solution is unique and satisfy the matching conditions.

\section{INTERNAL CHARACTERISTIC LAYERS}

Let us consider the data (2.29) of Section 2.4. To fix ideas we shall suppose that $\Gamma_{0}$ contains the boundaries $x_{1}=0$ and $x_{1}=\pi$. It appears, according to (2.30), that the solution of the limit problem exhibits a discontinuity along $x_{2}=\gamma$. Moreover, we saw in example 2.2 that $f \notin V_{a}^{\prime}$.

By comparison with example 2.3 the problem addressed in the present section is thus analogous to that of the Section 5.1 but in a layer near $x_{2}=\gamma$. Instead of (4.1), the inner variable is now

$$
y_{2}=\frac{x_{2}-\gamma}{\eta}
$$

The domain $D$ is presently $D=(0, \pi) \times(-\infty,+\infty)$, consequently we shall have two matchings for $y_{2} \rightarrow \pm \infty$.

As in Section 5.1, the inner expansion is (5.1) with $\theta(\eta)=\eta^{-1}$. The analogue of equation (5.4) is

$$
\int_{D}\left\{\partial_{1}^{2} U_{1}^{0} V_{1}^{0}+D_{2}^{3} U_{1}^{0} D_{2}^{3} V_{1}^{0}\right\} \mathrm{d} x_{1} \mathrm{~d} y_{2}=\int_{D^{+}} \varphi\left(x_{1}\right) D_{2} V_{1}^{0} \mathrm{~d} x_{1} \mathrm{~d} y_{2}
$$

where $D^{+}=(0, \pi) \times(0,+\infty)$ (analogously $\left.D^{-}=(0, \pi) \times(-\infty, 0)\right)$. 
In each region $D^{+}$and $D^{-}$we again obtain equation (5.6) and the boundary conditions (taking on $x_{2}=\gamma$ the quantities $V_{1}^{0}, D_{2} V_{1}^{0}$ and $D_{2}^{2} V_{1}^{0}$ are continuous) become the interface conditions

$$
\left\{\left[\left|\begin{array}{c}
D_{2}^{3} U_{1}^{0}\left(x_{1}, 0\right)=0 \\
D_{2}^{4} U_{1}^{0}\left(x_{1}, 0\right)=0 \\
D_{2}^{5} U_{1}^{0}\left(x_{1}, 0\right)=\varphi\left(x_{1}\right)
\end{array}\right|\right]\right.
$$

where $[|\bullet|]$ denotes the jump across the interface. Obviously, we shall also prescribe

$$
\left[\left|U_{1}^{0}\right|\right]=\left[\left|D_{2} U_{1}^{0}\right|\right]=\left[\left|D_{2}^{2} U_{1}^{0}\right|\right]=0 .
$$

Moreover, the function $U_{1}^{0}$ satisfies the boundary conditions

$$
U_{1}^{0}\left(0, y_{2}\right)=U_{1}^{0}\left(\pi, y_{2}\right)=0
$$

and the matching conditions

$$
U_{1}^{0}\left(x_{1}, y_{2}\right) \underset{y_{2} \rightarrow \pm \infty}{\longrightarrow} 0
$$

As in Section 5.1, we search in each region $D^{+}$and $D^{-}$a solution of the form (5.8) with coefficients $a_{n}^{+}$and $a_{n}^{-}$ respectively which satisfy

$$
-a_{n}^{ \pm(6)}\left(y_{2}\right)+n^{2} a_{n}^{ \pm}\left(y_{2}\right)=0
$$

and are bounded in their corresponding domain of definition, so that

$$
\left\{\begin{array}{l}
a_{n}^{+}\left(y_{2}\right)=\sum_{k=2}^{4} c_{k}^{+} \exp \left[n^{\frac{1}{3}} \exp \left(\frac{i k \pi}{3}\right) y_{2}\right] \\
a_{n}^{-}\left(y_{2}\right)=\sum_{k=0}^{3} c_{k}^{-} \exp \left[n^{\frac{1}{3}} \exp \left(\frac{i(2 k+1) \pi}{3}\right) y_{2}\right] .
\end{array}\right.
$$

Moreover, they satisfy the interface conditions

$$
\left\{\begin{array}{l}
{\left[\left|a_{n}\right|\right]=\left[\left|a_{n}^{1}\right|\right]=\cdots=\left[\left|a_{n}^{(4)}\right|\right]=0} \\
{\left[\left|a_{n}^{(5)}\right|\right]=\Phi_{n}}
\end{array}\right.
$$

where $\Phi_{n}$ is the coefficient of $\sin n x_{1}$ in the Fourier expansion of the function $\varphi\left(x_{1}\right)$.

\section{The CHARACTERISTIC BOUndary LAYER REVISITED. LAGRANGE MUltiPlier AND BOUNDARY CONDITIONS}

We saw in Sections 4, 5 and 6 that the leading terms of the solutions in the layers satisfy the constraint (4.6), and accordingly the corresponding equations must involve a Lagrange multiplier. This was avoided in the previous study by taking test functions satisfying themselves the constraint (4.6) so that, taking into account this constraint, we obtained the equation satisfied by $U_{1}^{0}\left(x_{1}, y_{2}\right)$ and the corresponding boundary conditions. Clearly this amounts to eliminate $U_{2}^{0}\left(x_{1}, y_{2}\right)$ and the Lagrange multiplier. In order to exhibit the role of the multiplier in the equations and the boundary conditions we now consider again the case of Section 5.1 which is a characteristic layer along the free boundary $x_{2}=0$. So that we consider expansions (5.1)

$$
\left\{\begin{array}{l}
u_{1}^{\varepsilon}\left(x_{1}, x_{2}\right)=\eta^{-1} U_{1}^{\eta}\left(x_{1}, y_{2}\right)=\eta^{-1} U_{1}^{0}+U_{1}^{1}+\cdots \\
u_{2}^{\varepsilon}=\eta^{-2} U_{2}^{\eta}\left(x_{1}, y_{2}\right)=\eta^{-2} U_{2}^{0}+\eta^{-1} U_{2}^{1}+U_{2}^{2}+\cdots
\end{array}\right.
$$


which gives the new form of (5.2):

$$
\begin{aligned}
\int_{D}\left\{\partial_{1} U_{1}^{\eta} \partial_{1} V_{1}^{\eta}+\frac{1}{\eta^{2}}\left(D_{2} U_{1}^{\eta}-U_{2}^{\eta}\right)\left(D_{2} V_{1}^{\eta}-V_{2}^{\eta}\right)+D_{2}^{2} U_{2}^{\eta} D_{2}^{2} V_{2}^{\eta}+\cdots\right\} & \mathrm{d} x_{1} \mathrm{~d} y_{2} \\
& =\int_{D}\left[f_{2}\left(x_{1}, 0\right)+\cdots\right] V_{2}^{\eta} \mathrm{d} x_{1} \mathrm{~d} y_{2} .
\end{aligned}
$$

Taking as test functions $V_{1}^{\eta} \equiv 0, V_{2}$ independent of $\eta$ and by identifying to zero the terms of order $\eta^{-2}$ and $\eta^{-1}$ we obtain

$$
\begin{aligned}
& D_{2} U_{1}{ }^{0}-U_{2}{ }^{0}=0 \\
& D_{2} U_{1}{ }^{1}-U_{2}{ }^{1}=0 .
\end{aligned}
$$

In the sequel, it will prove useful to define the new unknown $p\left(x_{1}, y_{2}\right)$ by

$$
D_{2} U_{1}^{2}-U_{2}^{2}=p
$$

Now, taking $V_{1} V_{2}$ arbitrary and independent of $\eta$ in (7.2), on account of (7.3) and (7.4) at the leading order we have

$$
\int_{D}\left\{\partial_{1} U_{1}^{0} \partial_{1} V_{1}+p\left(D_{2} V_{1}-V_{2}\right)+D_{2}^{2} U_{2} D_{2}^{2} V_{2}\right\} \mathrm{d} x_{1} \mathrm{~d} y_{2}=\int_{D} f_{2}\left(x_{1}, 0\right) V_{2} \mathrm{~d} x_{1} \mathrm{~d} y_{2} .
$$

Taking $V_{1}$ and $V_{2}$ vanishing in neighbourhoods of $x_{1}=0$ and $x_{1}=\pi$, integration by parts give

$$
\begin{aligned}
\int_{D}\left\{\left(-\partial_{1}^{2} U_{1}^{0}-D_{2} p\right) V_{1}+\left(D_{2}^{4} U_{2}^{0}-p\right) V_{2}\right\} \mathrm{d} x_{1} \mathrm{~d} y_{2} \\
\quad+\int_{\Gamma_{1}}\left\{-p V_{1}+D_{2}^{3} U_{2}^{0} V_{2}-D_{2}^{2} U_{2}^{0} D_{2} V_{2}\right\}\left(x_{1}, 0\right) d x_{1}=\int_{D} f_{2}\left(x_{1}, 0\right) V_{2} \mathrm{~d} x_{1} \mathrm{~d} y_{2}
\end{aligned}
$$

from which we obtain the system of equations

$$
\left\{\begin{array}{l}
-\partial_{1}^{2} U_{1}^{0}-D_{2} p=0 \\
D_{2}^{4} U_{2}^{0}-p=f_{2}\left(x_{1}, 0\right)
\end{array}\right.
$$

and, as $V_{1}, V_{2}$ and $D_{2} V_{2}$ are arbitrary on $\Gamma_{1}$, we have the natural boundary conditions

$$
\left\{\begin{array}{l}
-p\left(x_{1}, 0\right)=0 \\
D_{2}^{3} U_{2}^{0}\left(x_{1}, 0\right)=0 \\
-D_{2}^{2} U_{2}^{0}\left(x_{1}, 0\right)=0 .
\end{array}\right.
$$

Moreover, $U_{1}^{0}$ satisfies the principal boundary conditions

$$
U_{1}^{0}\left(0, y_{2}\right)=U_{1}^{0}\left(\pi, y_{2}\right)=0
$$

and the matching conditions

$$
\left\{\begin{array}{c}
U_{1}^{0}\left(x_{1}, y_{2}\right) \underset{y_{1} \rightarrow+\infty}{\longrightarrow} 0 \\
U_{2}^{0}\left(x_{1}, y_{2}\right) \underset{y_{1} \rightarrow+\infty}{\longrightarrow} 0
\end{array}\right.
$$


Problem (7.5)-(7.9) determines the unknowns $U_{1}^{0}, U_{2}^{0}$ and $p$, it constitutes the boundary layer problem with Lagrange multiplier. We should immediately verify that by eliminating $p$ and $U_{2}^{0}$ we obtain the equation (5.6) and the boundary conditions (5.7).

Remark 2. It should be easily seen that, on account of expansions (7.1), of (7.3) and (7.4) and of the definition (7.5) of $p$, the equations (7.6) and the boundary conditions (7.7) are respectively the leading order terms of (1.6) and (1.7) with $F_{1}=F_{2}=\mathcal{C}=0$.

Remark 3. The solution $\left(U_{1}^{0}, U_{2}^{0}\right)$ was obtained using two different methods. In the first one, the test functions were taken satisfying the constraint and the equation was obtained with $V_{1}$ arbitrary (but not $V_{2}$ ). On the other hand, in the second method, we took $V_{1}$ and $V_{2}$ arbitrary so that the corresponding problem involved a new unknown $p$ which is the Lagrange multiplier of the problem. Indeed, denoting by $B$ the operator defined by

$$
\left(u_{1}, u_{2}\right) \stackrel{B}{\longmapsto} D_{2} u_{1}-u_{2}
$$

which defines the constraint (7.3), the corresponding adjoint $B^{*}$ is defined by

$$
B^{*} p=\left(-D_{2} p,-p\right)^{t}
$$

which are precisely the terms in $p$ in system (7.6). Then we have the classical structure of a constrained problem with Lagrange multiplier [2]. Moreover, it is known that in penalty problems of the form (7.2) the Lagrange multiplier $p$ of the limit problem is

$$
p=\lim _{\eta \rightarrow 0} \frac{1}{\eta^{2}}\left(D_{2} U_{1}^{\eta}-U_{2}^{\eta}\right)
$$

that agrees with our results (see (7.1), (7.3), (7.4) and (7.5)).

\section{Boundary LAYER ALONG A NON-CHARACTERISTIC PART OF $\Gamma_{0}$}

We saw in Section 3.2 that the thickness of a non-characteristic layer is of order

$$
\eta=\varepsilon^{\frac{1}{2}}
$$

and the scaling of $u^{\varepsilon}$ is such that $u_{2}^{\varepsilon} / u_{1}^{\varepsilon}=\mathcal{O}\left(\eta^{-1}\right)=\mathcal{O}\left(\varepsilon^{-\frac{1}{2}}\right)$. To fix ideas, let us consider the non-characteristic layer along $x_{1}=0$ which is supposed to belong to $\Gamma_{0}$. Then we tentatively search for asymptotic expansions of the form

$$
\left\{\begin{array}{l}
u_{1}^{\varepsilon}\left(x_{1}, x_{2}\right)=\eta U_{1}^{\eta}\left(y_{1}, x_{2}\right)=\eta\left[U_{1}^{0}+\eta U_{1}^{1}+\cdots\right] \\
u_{2}^{\varepsilon}=U_{2}^{\eta}\left(y_{1}, x_{2}\right)=U_{2}^{0}+\eta U_{2}^{1}+\cdots
\end{array}\right.
$$

where

$$
y_{1}=\frac{x_{1}}{\eta}
$$

In the sequel, we shall denote by $D_{1}$ the derivative with respect to $y_{1}$ :

$$
D_{1}=\eta \partial_{1}
$$

The expansions (8.2) hold true in the domain $D$ defined by $y_{1} \in(0,+\infty), x_{2} \in(0, \pi)$. 
The solution $U^{\eta}$ must satisfy the boundary conditions

$$
U_{1}^{\eta}\left(0, x_{2}\right)=U_{2}^{\eta}\left(0, x_{2}\right)=D_{1} U_{2}^{\eta}\left(0, x_{2}\right)=0
$$

and the matching conditions for $y_{1} \rightarrow+\infty$, which we shall consider later.

The variational formulation hold true in the domain $D$, at least for test functions with compact support in $y_{1}$, we shall take them depending on $\eta$ in a form analogous to (8.2). Consequently, we have

$$
\begin{gathered}
\int_{D}\left\{D_{1} U_{1}^{\eta} D_{1} V_{1}^{\eta}+\left(\eta \partial_{2} U_{1}^{\eta}-U_{2}^{\eta}\right)\left(\eta \partial_{2} V_{1}^{\eta}-V_{2}^{\eta}\right)+\frac{\varepsilon^{2}}{\eta^{4}}\left[D_{1}^{2} U_{2}^{\eta} D_{1}^{2} V_{2}^{\eta}+\cdots\right]\right\} \mathrm{d} x_{1} \mathrm{~d} y_{2} \\
=\int_{D}\left\{\left[f_{1}\left(0, x_{2}\right)+\cdots\right] \eta V_{1}^{\eta}+\left[f_{2}\left(0, x_{2}\right)+\eta y_{1} \partial_{1} f_{2}\left(0, x_{2}\right)+\cdots\right] V_{2}^{\eta}\right\} \mathrm{d} y_{1} \mathrm{~d} x_{2}
\end{gathered}
$$

where $f$ is supposed to be smooth and was represented by its Taylor expansion in the vicinity of $x_{1}=0$. We note that, according to $(8.1), \varepsilon^{2} / \eta^{4}=1$ in (8.6).

Taking $V_{1}^{\eta} \in \mathcal{D}(D)$ and $V_{2}^{\eta} \equiv 0$ in (8.6), the leading term (of order $\mathcal{O}(1)$ ) gives

$$
D_{1}^{2} U_{1}^{0}=0
$$

so that

$$
U_{1}^{0}\left(y_{1}, x_{2}\right)=A\left(x_{2}\right) y_{1}+B\left(x_{2}\right)
$$

Conditions (8.5) give $B\left(x_{2}\right)=0$ and the matching condition writes

$$
A\left(x_{2}\right)=\partial_{1} u_{1}^{0}\left(0, x_{2}\right)
$$

where $u_{1}^{0}\left(x_{1}, x_{2}\right)$ is the corresponding solution of the limit problem $P(0)$.

On other hand, if we take $V_{1}^{\eta} \equiv 0$ and $V_{2}^{\eta} \in \mathcal{D}(D)$, then the leading term (of order $\mathcal{O}(1)$ ) gives

$$
D_{1}^{4} U_{2}^{0}+U_{2}^{0}=f_{2}\left(0, x_{2}\right)
$$

The solution of (8.10) may be written under the form

$$
U_{2}^{0}=\tilde{U}_{2}^{0}+\hat{U}_{2}^{0}\left(x_{2}\right)
$$

where

$$
\hat{U}_{2}^{0}\left(x_{2}\right)=f_{2}\left(0, x_{2}\right)
$$

then $\tilde{U}_{2}^{0}$ is the bounded solution of

$$
D_{1}^{4} \tilde{U}_{2}^{0}+\tilde{U}_{2}^{0}=0
$$

which is a differential equation in $y_{1}$ with parameter $x_{2}$, the bounded solution of which is

$$
\tilde{U}_{2}^{0}\left(y_{1}, x_{2}\right)=\sum_{k=1}^{2} c_{k}\left(x_{2}\right) \exp \left(e^{\frac{(2 k+1) i \pi}{4}} y_{1}\right)
$$


It satisfies the conditions:

$$
\lim _{y_{1} \rightarrow+\infty} \tilde{U}_{2}^{0}\left(y_{1}, x_{2}\right)=f_{2}\left(0, x_{2}\right)
$$

which is the matching condition with the corresponding solution of the limit problem $P(0)$ of Section 2 .

Otherwise this solution must satisfy the boundary conditions (8.5) which gives

$$
\left\{\begin{array}{l}
c_{1}\left(x_{2}\right)+c_{2}\left(x_{2}\right)=f_{2}\left(0, x_{2}\right) \\
c_{1}\left(x_{2}\right) e^{\frac{3 i \pi}{4}}+c_{2}\left(x_{2}\right) e^{-\frac{3 i \pi}{4}}=0
\end{array}\right.
$$

which determine the functions $c_{k}\left(x_{2}\right)$.

\section{The Distributional solutions of $P(0)$ AS Limits of internal LAYERS}

We saw in Section 2.4 that the solutions of the limit problem $P(0)$ exhibit singular terms when the datum $f$ is not smooth with respect to $x_{2}$. In the particular case when $f$ is defined by (2.29) we considered in Section 6 the corresponding internal layer. We admit that $\Gamma_{0}$ contains the two vertical boundary layers. In this section we are showing that the singular terms (containing $\delta_{\gamma}$ and $\delta_{\gamma}^{\prime}$ ) in (2.30) are limits of the corresponding terms in the layers. In the sequel, we only consider the component $u_{1}$ as the study of $u_{2}$ is analogous and even follows from that of $u_{1}$.

In order to write explicitly $(2.30)_{1}$, let us consider the Fourier sinus expansion of $\varphi$ :

$$
\varphi\left(x_{1}\right)=\sum_{n=1}^{+\infty} A_{n} \sin n x_{1} .
$$

As in the present case $A$ is the Laplace operator on $(0, \pi)$ with Dirichlet boundary conditions, we have

$$
\left(A^{-1} \varphi\right)\left(x_{1}\right)=-\sum_{n=1}^{+\infty} \frac{A_{n}}{n^{2}} \sin n x_{1}
$$

so that $(2.30)_{1}$ takes the form

$$
u_{1}\left(x_{1}, x_{2}\right)=\left(-\sum_{n=1}^{+\infty} \frac{A_{n}}{n^{2}} \sin n x_{1}\right) \delta\left(x_{2}-\gamma\right) .
$$

It follows from Section 6 that the inner expansion of $u_{1}^{\varepsilon}$ in the internal layer is

$$
u_{1}^{\varepsilon}\left(x_{1}, x_{2}\right) \cong \frac{1}{\eta} U_{1}^{0}\left(x_{1}, \frac{x_{2}-\gamma}{\eta}\right)+\cdots
$$

The aim of this section is to prove that

$$
u_{1}\left(x_{1}, x_{2}\right)=\lim _{\eta \rightarrow 0} \frac{1}{\eta} U_{1}^{0}\left(x_{1}, \frac{x_{2}-\gamma}{\eta}\right) .
$$

From elementary distribution theory it follows that the right-hand side of (9.5) is

$$
\left(\int_{-\infty}^{+\infty} U_{1}^{0}\left(x_{1}, y_{2}\right) \mathrm{d} y_{2}\right) \delta\left(x_{2}-\gamma\right)
$$


where $x_{1}$ is a parameter. Consequently (9.5) amounts to prove that

$$
\int_{-\infty}^{+\infty} U_{1}^{0}\left(x_{1}, y_{2}\right) \mathrm{d} y_{2}=-\sum_{n=1}^{+\infty} \frac{A_{n}}{n^{2}} \sin n x_{1} .
$$

The function $U_{1}^{0}$ is the unique solution of (5.6) in each region $D^{+}$and $D^{-}$with the boundary and transmission conditions (6.3)-(6.5). It appears that $U_{1}^{0}$ is even with respect to $y_{2}$ as it coincides with the function obtained by changing $y_{2}$ into $-y_{2}$. Consequently, we may only consider the solution in the region $D^{+}$where $y_{2}>0$ which satisfies (5.6) and the boundary conditions

$$
\left\{\begin{array}{l}
D_{2} U_{1}^{0}\left(x_{1}, 0\right)=0 \\
D_{2}^{(3)} U_{1}^{0}\left(x_{1}, 0\right)=0 \\
D_{2}^{(5)} U_{1}^{0}\left(x_{1}, 0\right)=\frac{\varphi\left(x_{1}\right)}{2}
\end{array}\right.
$$

and (6.4) as well as the matching conditions

$$
U_{1}^{0}\left(x_{1}, y_{2}\right) \underset{y_{2} \rightarrow+\infty}{\longrightarrow} 0 .
$$

It follows from $(6.7)_{1}$ that $U_{1}^{0}$ writes

$$
U_{1}^{0}\left(x_{1}, y_{2}\right)=\sum_{n=1}^{+\infty} \sum_{k=2}^{4} C_{n k} \sin n x_{1} \exp \left[n^{\frac{1}{3}} e^{\frac{i k \pi}{3}} y_{2}\right] .
$$

The boundary conditions (9.7) give

$$
\left\{\begin{array}{l}
-e^{-\frac{i \pi}{3}} C_{n 2}-C_{n 3}-e^{\frac{i \pi}{3}} C_{n 4}=0 \\
C_{n 2}-C_{n 3}+C_{n 4}=0 \\
-e^{\frac{i \pi}{3}} C_{n 2}-C_{n 3}-e^{-\frac{i \pi}{3}}=\frac{A_{n}}{2 n^{\frac{5}{3}}}
\end{array}\right.
$$

As $U_{1}^{0}$ is even, the left-hand side of $(9.6)$ is

$$
\begin{aligned}
2 \int_{0}^{+\infty} U_{1}^{0}\left(x_{1}, y_{2}\right) \mathrm{d} y_{2} & =2 \sum_{n=1}^{+\infty} \sum_{k=2}^{4} C_{n k} \sin n x_{1} \int_{0}^{+\infty} \exp \left[n^{\frac{1}{3}} e^{\frac{i k \pi}{3}} y_{2}\right] \mathrm{d} y_{2} \\
& =-2 \sum_{n=1}^{+\infty} \sum_{k=2}^{4} C_{n k} \sin n x_{1} \frac{1}{n^{\frac{1}{3}}} e^{-\frac{i k \pi}{3}}=2 \sum_{n=1}^{+\infty} \frac{\sin n x_{1}}{n^{\frac{1}{3}}}\left(C_{n 2} e^{\frac{i \pi}{3}}+C_{n 3}+C_{n 4} e^{-\frac{i \pi}{3}}\right) \\
& =-\sum_{n=1}^{+} \frac{A_{n}}{n^{2}} \sin n x_{1}
\end{aligned}
$$

(where relation $(9.9)_{3}$ was used) which proves (9.6).

\section{NumERICAL EXPERIMENTS}

In this section we present numerical experiments concerning the problem $P(\varepsilon)$ in several cases of boundary conditions and loading. The numerical computations are implemented with reduced Hermite finite elements. An exact numerical integration of the rigidity matrices needs twelve Gauss points. 


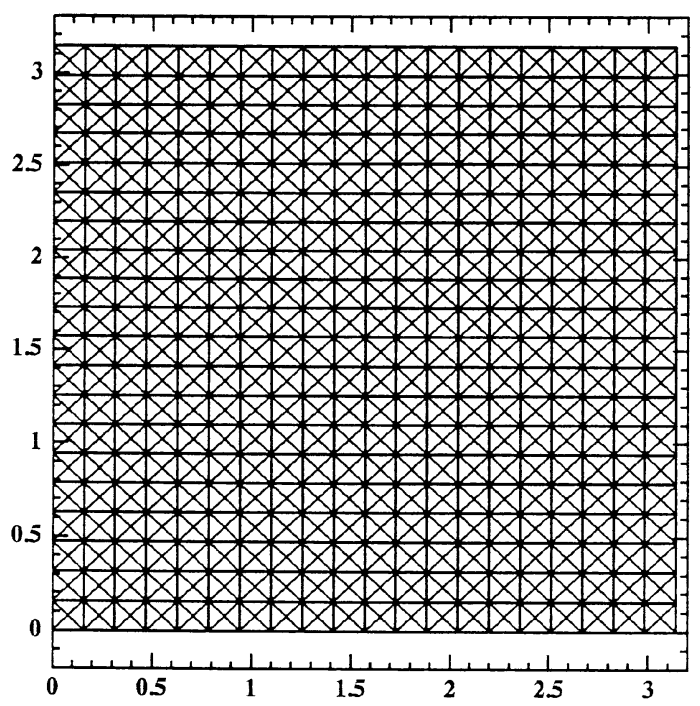

FIGURE 1. Uniform mesh.

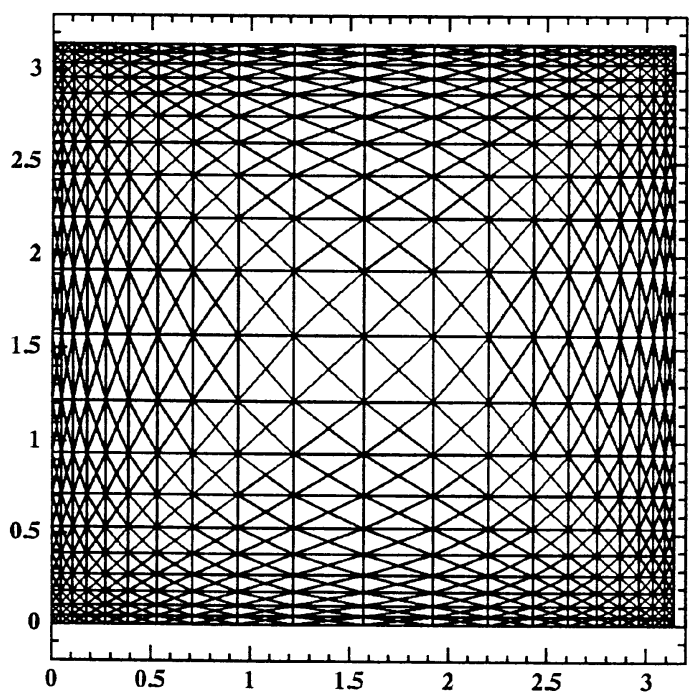

FigurE 2. Adapted mesh for characteristic boundary layers.

The meshes for the domain $\Omega=[0, \pi] \times[0, \pi]$ are generated using the Modulef code. The mesh is obtained by symmetry from the basic square $[0, \pi / 2] \times[0, \pi / 2]$, a first time around $x_{1}=\pi / 2$ and then around $x_{2}=\pi / 2$. This device allows us to perform easily the refinement of the mesh in the vicinity of the boundary layers. In the sequel we shall compare the results obtained with an uniform mesh and a non uniform one refined in the vicinity of the boundary layers. In the uniform mesh, the basic square is divided in $N \times M$ rectangles where the parameters $N$ and $M$ correspond to the number of divisions along the axes $x_{1}$ and $x_{2}$ respectively. Each rectangle is then divided in four triangles. Figure 1, shows the uniform mesh generated in this way. The non uniform mesh (see Fig. 2) is generated in the same way but using a function of distribution of the points according to a geometric progression with ratio $q$. 


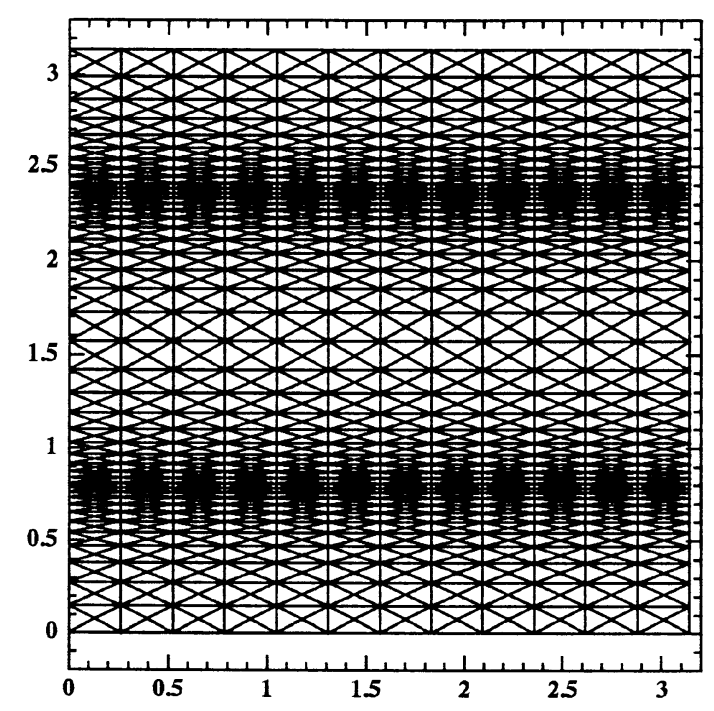

Figure 3. Adapted mesh for internal layers.

In the case of internal layers we analogously get the mesh shown in Figure 3.

\subsection{Totally clamped boundary}

We consider the problem $P(\varepsilon)$ for $\varepsilon=0.001$ and $\vec{f}=\left(f_{1}, f_{2}\right)=\left(0, x_{2}-1\right)$. We shall focus our attention on the characteristic boundary layers along $x_{2}=0$ and $x_{2}=\pi$ (see Sect. 4). These layers are more important than the non characteristic ones along $x_{1}=0$ and $x_{1}=\pi$, compare (4.2) with $\eta=\varepsilon^{\frac{1}{3}}$ and (8.2) with $\eta=\varepsilon^{\frac{1}{2}}$.

In the characteristic layers the order of the thickness is $\eta=0.1$. Computations with the uniform mesh (Fig. 1) give nearly good results with $N=M=11$, i.e. with mesh step $h=\pi / 21$ in both directions; the values of $u_{2}^{\varepsilon}$ are shown in Figure 4. With the non uniform mesh (Fig. 2), we observe that the mesh may be coarse provided that the layer region is sufficiently covered, we took $N=7, M=11, q=1.4$ so that the first four steps are contained in the length $\eta$; the values of $u_{2}^{\varepsilon}$ are shown in Figure 5 . The first and the second meshes contain 1600 and 960 triangular elements respectively. In the present case the advantage of the adapted mesh is not very significant.

\subsection{Case of a characteristic free boundary with $f_{2} \neq 0$}

The problem $P(\varepsilon)$ is again considered with the previous data $\varepsilon$ and $\vec{f}$. The boundary $x_{2}=0$ is free and the rest of the boundary is clamped. We observe that $f_{2}$ does not vanish on the free boundary so that in this case the corresponding boundary layer is (5.1) with $\theta(\eta)=\eta^{-1}=\varepsilon^{-\frac{1}{3}}$ (see (5.3)). We observe that $u_{2}^{\varepsilon}$ is of order $\eta^{-2}=\varepsilon^{-\frac{2}{3}}$ whereas in the previous case it was of order $\varepsilon^{-\frac{1}{3}}$. The magnitude of the boundary layer in the present case leads to a significant advantage of the adapted mesh.

Taking the uniform mesh, the mesh step must be chosen sufficiently small to get a satisfactory result. In fact we must take at least $N=M=31$ so that the mesh step is $h=\pi / 61$ which corresponds to 14400 triangular elements.

When using a non uniform mesh, satisfactory results are obtained with the same mesh as in the previous subsection which correspond to 960 triangular elements.

The numerical results for $u_{2}^{\varepsilon}$ in both cases are shown in Figures 6 and 7, they are obtained in 15 minutes with the uniform mesh and only 2 minutes with the adapted mesh. 


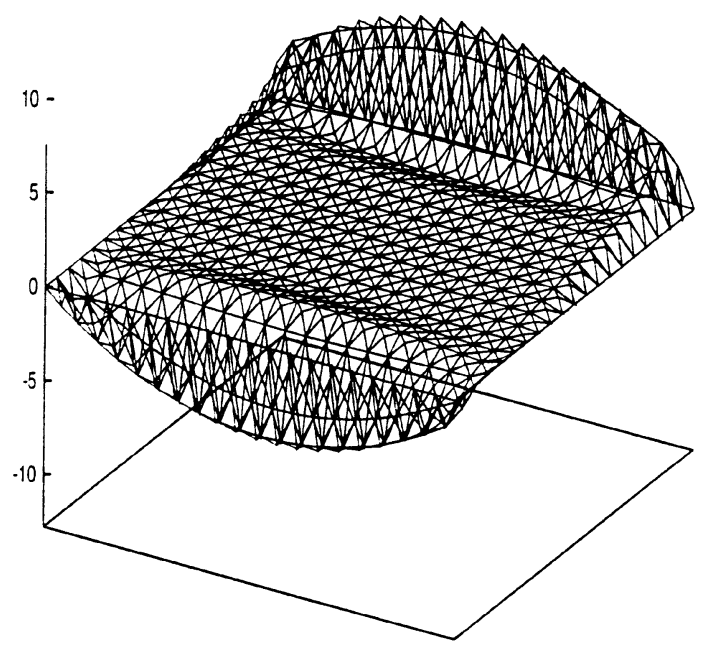

FIGURE 4. Three dimensional plot of $U_{2}$ (uniform mesh).

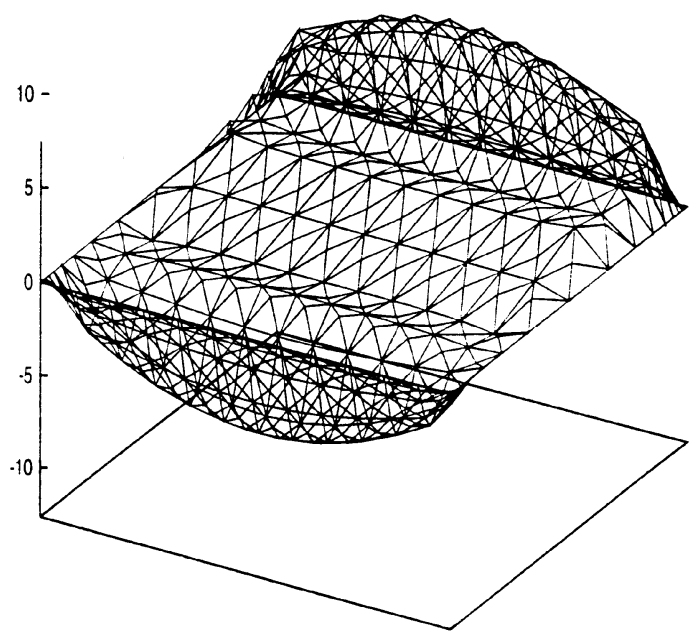

Figure 5. Three dimensional plot of $U_{2}$ (adapted mesh).

\subsection{Internal layers}

We now consider the problem $P(\varepsilon)$ with the whole boundary clamped, $\varepsilon=0.001$ and

$$
\vec{f}=\left\{\begin{array}{l}
f_{1}=0 \\
f_{2}=\left\{\begin{array}{l}
1 \text { for } x_{2}>\pi / 2 \\
0 \text { for } x_{2}<\pi / 2
\end{array}\right.
\end{array}\right.
$$

so that the loading has a discontinuity along the characteristic $x_{2}=\frac{\pi}{2}$. According to the results of Section 6 , the scaling is the same as in the previous subsection. We present in Figure 8 the numerical results obtained with the same uniform mesh as before. Obviously, we observe the presence of an internal layer along the characteristic $x_{2}=\pi / 2$. 


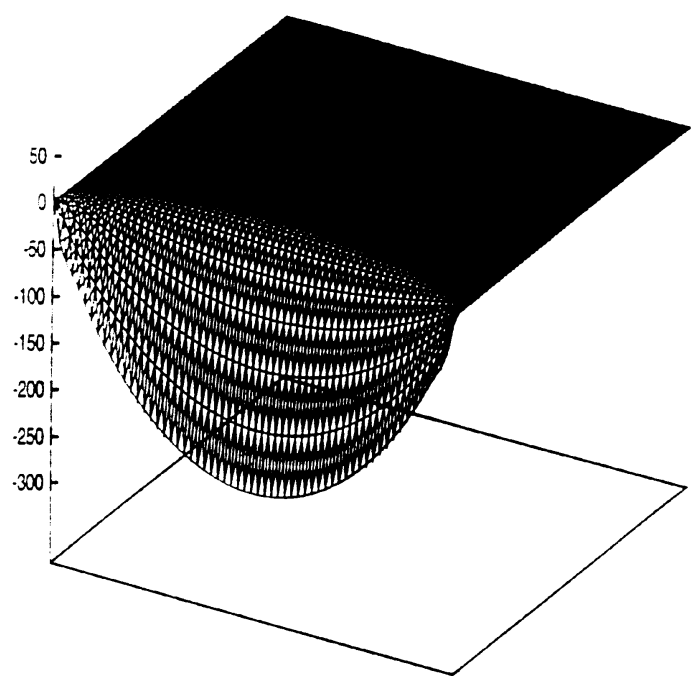

FiguRE 6. Three dimensional plot of $U_{2}$ (uniform mesh).

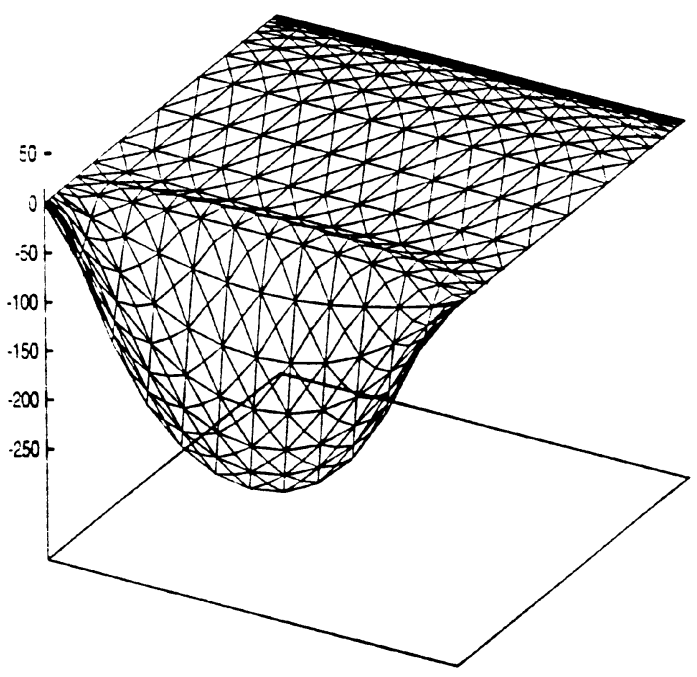

Figure 7 . Three dimensional plot of $U_{2}$ (adapted mesh).

In order to exhibit the propagation of the singularities along the characteristics evoked in Section 2.5 we consider instead of (10.1) the loading

$$
\vec{f}=\left\{\begin{array}{l}
f_{1}=0 \\
f_{2}=\left\{\begin{array}{l}
1 \text { on }\left[0, \frac{\pi}{2}\right] \times\left[\frac{\pi}{2}, \pi\right] \\
0 \text { elsewhere }
\end{array}\right.
\end{array}\right.
$$

The numerical results for $u_{2}^{\varepsilon}$ obtained with the non uniform mesh $N=7, M=11, q=0.7$ are shown in Figure 9 . We observe an internal layer along the whole characteristic $x_{2}=\pi / 2$ whereas the discontinuity of the data are 


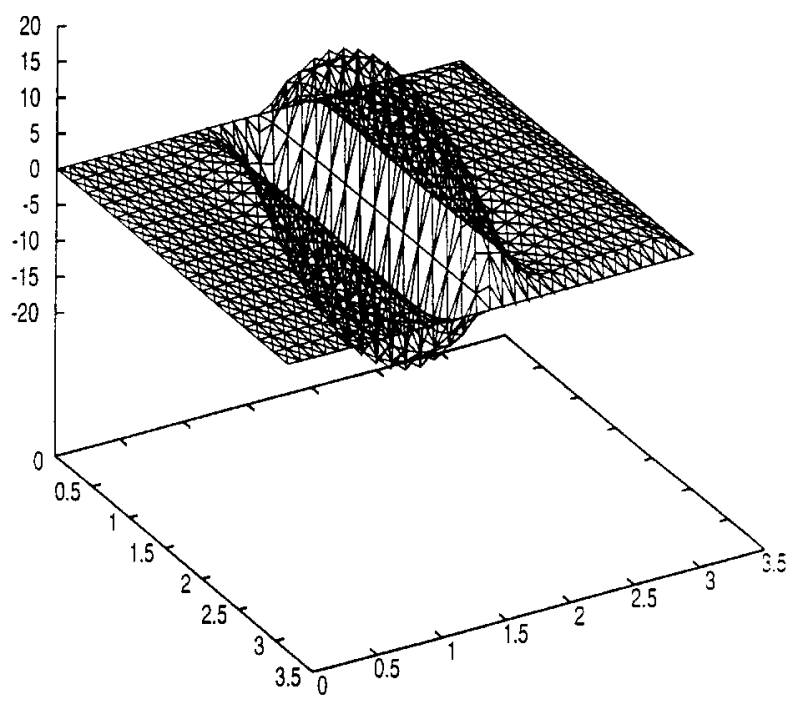

FIGURE 8. Three dimensional plot of $U_{2}$ for uniform unit upper rectangle loading (uniform mesh).

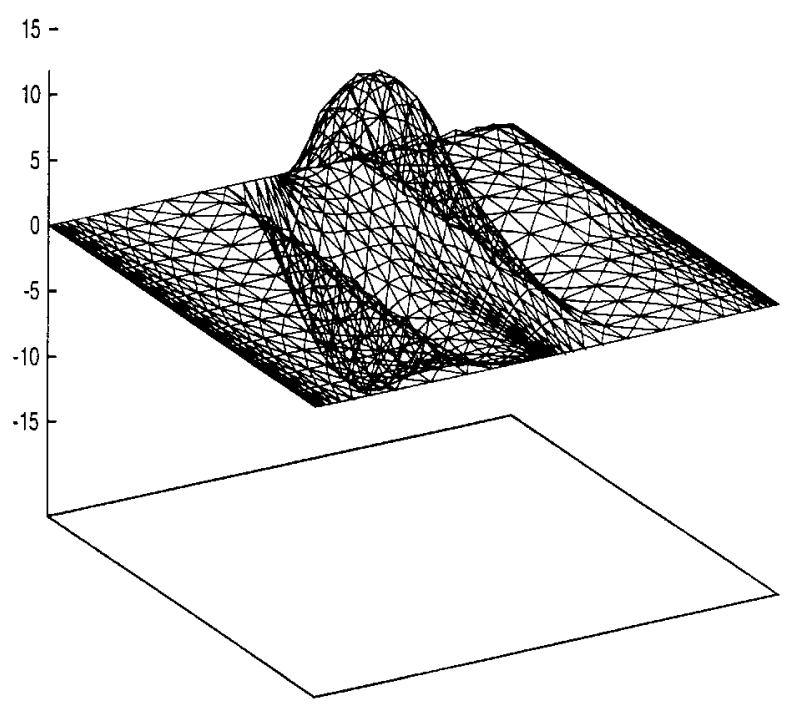

FigurE 9. Three dimensional plot of $U_{2}$ for uniform unit square loading (adapted mesh).

confined in the interval $0<x_{1}<\pi / 2$. This fact is even more explicit in Figure 10 which represents $u_{2}^{\varepsilon}$ along the section $x_{1}=3 \pi / 4$.

Another interesting example of propagation of the singularities is given Figure 11 where the loading is chosen as follows

$$
\vec{f}=\left\{\begin{array}{l}
f_{1}=0 \\
f_{2}=\left\{\begin{array}{l}
1 \text { on } \Delta=\left\{x_{1} \in\left[\frac{\pi}{4}, \frac{3 \pi}{4}\right],\left|x_{2}+x_{1}-\pi\right| \leq 0.1\right\} \\
0 \text { elsewhere. }
\end{array}\right.
\end{array}\right.
$$




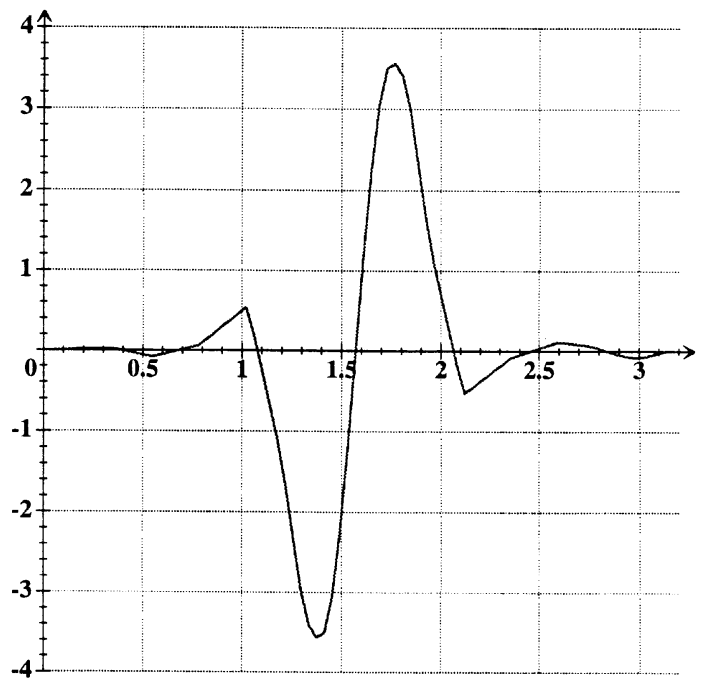

Figure 10. Graph of $U_{2}$ along the cross section $x=3 \pi / 4$ for unif.

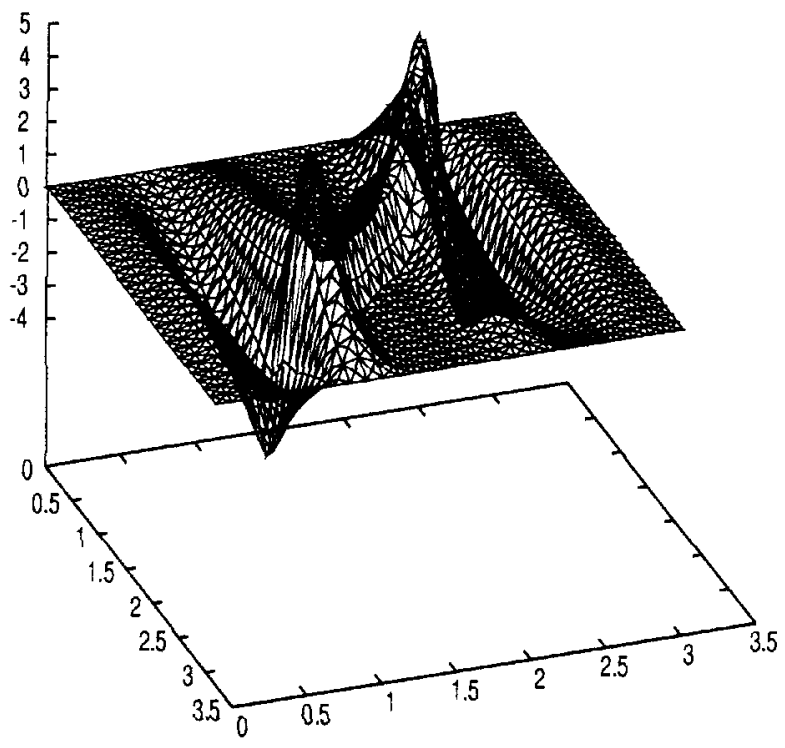

FiguRE 11. Three dimensional plot of $U_{2}$ for internal layer with propagation (adapted mesh).

We observe the presence of internal layers along the characteristics $x_{2}=\pi / 4$ and $x_{2}=3 \pi / 4$ which correspond to the extremities of the thin region $\Delta$, the cross-section $x_{1}=3 \pi / 4$ is represented in Figure 12 .

The numerical results for $u_{2}^{\varepsilon}$ are obtained using the adapted mesh with parameters $q=1, N=7$ and $M=11$ (which correspond to the mesh steps $h_{x_{1}}=\pi / 13$ and $h_{x_{2}}=\pi / 21$ in the directions $x_{1}$ and $x_{2}$ ) respectively. 


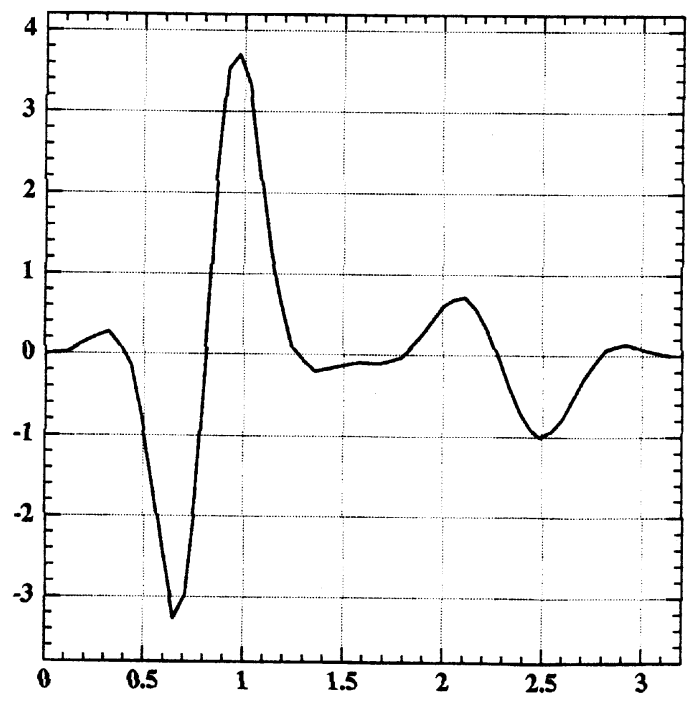

Figure 12. Graph of $U_{2}$ along the cross section $x=3 \pi / 4$.

TABLE 1

\begin{tabular}{|c|c|c|}
\hline Layer & Behavior & Energy \\
\hline $\begin{array}{c}\text { Characteristic } \\
\text { thickness: } \eta(\varepsilon)=\varepsilon^{\frac{1}{3}}\end{array}$ & $\frac{v_{2}^{\varepsilon}}{v_{1}^{\varepsilon}}=\mathcal{O}\left(\eta^{-1}\right)$ & \\
\hline clamped & $\left\{\begin{array}{l}v_{1}^{\varepsilon} \cong U_{1}^{0}\left(x_{1}, y_{2}\right) \\
v_{2}^{\varepsilon} \cong \eta^{-1} U_{2}^{0}\left(x_{1}, y_{2}\right)\end{array}\right.$ & $E_{C}=\mathcal{O}(\eta)$ \\
\hline $\begin{array}{c}\text { free } \\
f_{2}\left(x_{1}, 0\right)=0\end{array}$ & $\left\{\begin{array}{l}v_{1}^{\varepsilon} \cong U_{1}^{0}\left(x_{1}, y_{2}\right) \\
v_{2}^{\varepsilon} \cong \eta^{-1} U_{2}^{0}\left(x_{1}, y_{2}\right)\end{array}\right.$ & $E_{C}=\mathcal{O}(\eta)$ \\
\hline $\begin{array}{c}\text { free } \\
f_{2}\left(x_{1}, 0\right) \neq 0\end{array}$ & $\left\{\begin{array}{l}v_{1}^{\varepsilon} \cong \eta^{-1} U_{1}^{0}\left(x_{1}, y_{2}\right) \\
v_{2}^{\varepsilon} \cong \eta^{-2} U_{2}^{0}\left(x_{1}, y_{2}\right)\end{array}\right.$ & $E_{C}=\mathcal{O}\left(\eta^{-1}\right)$ \\
\hline $\begin{array}{l}\text { Non-characteristic } \\
\text { thickness: } \eta(\varepsilon)=\varepsilon^{\frac{1}{2}}\end{array}$ & $\begin{array}{c}\frac{v_{2}^{\varepsilon}}{v_{1}^{\varepsilon}}=\mathcal{O}\left(\eta^{-1}\right) \\
\left\{\begin{array}{l}v_{1}^{\varepsilon} \cong \eta U_{1}^{0}\left(x_{1}, y_{2}\right) \\
v_{2}^{\varepsilon} \cong U_{2}^{0}\left(x_{1}, y_{2}\right)\end{array}\right.\end{array}$ & $E=\mathcal{O}(\eta)$ \\
\hline $\begin{array}{l}\text { Internal Characteristic } \\
\text { thickness: } \eta(\varepsilon)=\varepsilon^{\frac{1}{3}} \\
\text { with discontinuity of } f_{2} \\
\text { on } x_{2}=0\end{array}$ & $\begin{array}{c}\frac{v_{2}^{\varepsilon}}{v_{1}^{\varepsilon}}=\mathcal{O}\left(\eta^{-1}\right) \\
\left\{\begin{array}{c}v_{1}^{\varepsilon} \cong \eta^{-1} U_{1}^{0}\left(x_{1}, y_{2}\right) \\
v_{2}^{\varepsilon} \cong \eta^{-2} U_{2}^{0}\left(x_{1}, y_{2}\right)\end{array}\right.\end{array}$ & $E_{C}=\mathcal{O}\left(\eta^{-1}\right)$ \\
\hline
\end{tabular}

\section{Conclusions}

The main results of this paper are summed up in Table 1 which gives the order of the thickness $\eta$ and of the two components of $u$. The energy in the layer is also reported in Table 1. 
It appears that the energy tends to infinity in two cases: free characteristic boundary with $f_{2} \neq 0$ on it and internal layer bearing a discontinuity of $f_{2}$ on a characteristic. The energy in the layer tends to infinity as $\varepsilon$ tends to zero. Obviously, the energy in a region out of the layers is of order unity so that the above mentioned result agrees with Theorem 2.2. But the asymptotic structure also shows that the energy is asymptotically concentrated in the layers (but this is probably associated with the very definition of layers!). Finally, it should be pointed out that layers cutting the characteristics $x_{2}=$ const. at angle $\neq \pi / 2$ are analogous to the layers along $x_{1}=$ const. considered above. This is the reason why all these layers are called "non characteristic".

\section{REFERENCES}

[1] M. Bernadou, Méthodes d'éléments finis pour les problèmes de coques minces. Masson, Paris (1994).

[2] F. Brezzi and F. M. Fortin, Mixed and hybrid finite elements methods. Springer (1991).

[3] D. Choï, F.J. Palma, É. Sanchez Palencia and M.A. Vilariño, Remarks on membrane locking in the finite element computation of very thin elastic shells. Math. Modell. Num. Anal. 32 (1998) 131-152.

[4] P.G. Ciarlet, Mathematical elasticity, Vol. III, Theory of shells. North Holland, Amsterdam (to appear).

[5] D. Chapelle and K.J. Bathe, Fundamental considerations for the finite element analysis of shell structures, Computers and Structures 66 (1998) 19-36.

[6] P. Gérard and É. Sanchez Palencia, Sensitivity phenomena for certain thin elastic shells with edges. Math. Meth. Appl. Sci. (to appear).

[7] A.L. Goldenveizer, Theory of elastic thin shells. Pergamon, New York (1962).

[8] P. Karamian, Nouveaux résultats numériques concernant les coques minces hyperboliques inhibées: cas du paraboloïde hyperbolique. C. R. Acad. Sci. Paris Sér. IIb 326 (1998) 755-760.

[9] P. Karamian, Réflexion des singularités dans les coques hyperboliques inhibées. C.R. Acad. Sci. Paris Sér. IIb 326 (1998) 609-614.

[10] P. Karamian, Coques élastiques minces hyperboliques inhibées : calcul du problème limite par éléments finis et non reflexion des singularités. Thèse de l'Universté de Caen (1999).

[11] D. Leguillon, J. Sanchez-Hubert and É. Sanchez Palencia, Model problem of singular perturbation without limit in the space of finite energy and its computation. C.R. Acad. Sci. Paris Sér. IIb 327 (1999) 485-492.

[12] J.L. Lions and É. Sanchez Palencia, Problèmes sensitifs et coques élastiques minces. in Partial Differential Equations and Functional Analysis, in memory of P. Grisvard (J. Céa, D. Chesnais, G. Geymonat, J.L. Lions Eds.), Birkhauser, Boston (1996) 207-220.

[13] J.L. Lions and É. Sanchez Palencia, Sur quelques espaces de la théorie des coques et la sensitivité, in Homogenization and applications to material sciences, Cioranescu, Damlamian, Doneto Eds., Gakkotosho, Tokyo (1995) $271-278$.

[14] A.E.H Love, A treatrise on the mathematical theory of elasticity, Reprinted by Dover, New-York (1944).

[15] J. Pitkaranta and É. Sanchez Palencia, On the asymptotic behavior of sensitive shells with small thickness. C.R. Acad. Sci. Paris Sér. IIb 325 (1997) 127-134.

[16] H.S. Rutten, Theory and design of shells on the basis of asymptotic analysis. Rutten and Kruisman, Voorburg (1973).

[17] J. Sanchez-Hubert and É. Sanchez Palencia, Introduction aux méthodes asymptotiques et à l'homogénéisation, Masson, Paris (1992).

[18] J. Sanchez-Hubert and É. Sanchez Palencia, Coques élastiques minces. Propriétés asymptotiques. Masson, Paris (1997).

[19] J. Sanchez-Hubert and É. Sanchez Palencia, Pathological phenomena in computation of thin elastic shells. Transactions Can. Soc. Mech. Engin. 22 (1998) 435-446.

[20] M. Van Dyke, Perturbation methods in fluid mechanics. Academic Press, New-York (1964). 